\title{
Cyclotron resonance of the magnetic ratchet effect and second harmonic generation in bilayer graphene
}

\author{
Narjes Kheirabadi, ${ }^{1}$ Edward McCann, ${ }^{1}$ and Vladimir I. Fal'ko ${ }^{2}$ \\ ${ }^{1}$ Physics Department, Lancaster University, Lancaster LA1 4YB, United Kingdom \\ ${ }^{2}$ National Graphene Institute, The University of Manchester, Manchester M13 9PL, United Kingdom
}

(Received 15 November 2017; published 13 February 2018)

\begin{abstract}
We model the magnetic ratchet effect in bilayer graphene in which a dc electric current is produced by an ac electric field of frequency $\omega$ in the presence of a steady in-plane magnetic field and inversion-symmetry breaking. In bilayer graphene, the ratchet effect is tunable by an external metallic gate which breaks inversion symmetry. For zero in-plane magnetic field, we show that trigonal warping and inversion-symmetry breaking are able to produce a large dc valley current, but not a nonzero total dc charge current. For the magnetic ratchet in a tilted magnetic field, the perpendicular field component induces cyclotron motion with frequency $\omega_{c}$ and we find that the dc current displays cyclotron resonance at $\omega_{c}=\omega$, although this peak in the current is actually smaller than its value at $\omega_{c}=0$. Second harmonic generation, however, is greatly enhanced by resonances at $\omega_{c}=\omega$ and $\omega_{c}=2 \omega$ for which the current is generally much larger than at $\omega_{c}=0$.
\end{abstract}

DOI: 10.1103/PhysRevB.97.075415

\section{INTRODUCTION}

Recently, there has been interest in the magnetic ratchet effect in two-dimensional electron systems such as semiconductor quantum wells [1-6], graphene [7-9], and bilayer graphene [10]. It is a nonlinear effect $[11,12]$ producing a dc electric current in response to ac laser light in the presence of a steady in-plane magnetic field and broken inversion symmetry. Here, we consider the magnetic ratchet in bilayer graphene [10,13-17] for which inversion asymmetry is tunable by applying a gate voltage [14,18-20], and we take into account a tilted magnetic field. If the magnetic field has a perpendicular component, the efficiency of the ratchet should be dramatically increased under cyclotron resonance conditions [6] when the cyclotron frequency $\omega_{c}$ is close to the ac field frequency $\omega$. For the dc current, we find that there is indeed a resonance at $\omega_{c}=\omega$, but, as a function of $\omega_{c}$, the dc current is actually larger at $\omega_{c}=0$; Fig. 1(a). For second harmonic generation [19-27], however, we find there are resonances at $\omega_{c}=\omega$ and $\omega_{c}=2 \omega$ which are generally much stronger than the current at $\omega_{c}=0$; Fig. 1(b).

We begin by summarizing our results. In bilayer graphene, the intraband contribution (which is relevant in the semiclassical regime $\epsilon_{F} \gg \hbar \omega$ where $\epsilon_{F}$ is the Fermi level) to the dc current density $\mathbf{J}^{(0)}$ is given by

$$
\begin{aligned}
\mathbf{J}^{(0) \approx} & \left\{\left[\left(\mathbf{B}_{\|} \times \hat{\mathbf{n}}_{z}\right) \cdot \mathbf{E}\right] \mathbf{E}^{*}+\left[\left(\mathbf{B}_{\|} \times \hat{\mathbf{n}}_{z}\right) \cdot \mathbf{E}^{*}\right] \mathbf{E}\right. \\
& \left.-\left(\mathbf{B}_{\|} \times \hat{\mathbf{n}}_{z}\right)|\mathbf{E}|^{2}\right\} \operatorname{Re}\left(M_{1}\right) \\
& +\left\{\mathbf{E}\left(\mathbf{B}_{\|} \cdot \mathbf{E}^{*}\right)+\mathbf{E}^{*}\left(\mathbf{B}_{\|} \cdot \mathbf{E}\right)\right. \\
& \left.-\mathbf{B}_{\|}|\mathbf{E}|^{2}\right\} \operatorname{Im}\left(M_{1}\right),
\end{aligned}
$$

for normally incident radiation with an in-plane alternating electric field $\mathbf{E}(t)$ of angular frequency $\omega$,

$$
\mathbf{E}(t)=\mathbf{E} e^{-i \omega t}+\mathbf{E}^{*} e^{i \omega t}
$$

and in-plane magnetic field $\mathbf{B}_{\|} ; \hat{\mathbf{n}}_{z}$ is a unit vector in the perpendicular $(z)$ direction. We include terms that are second order in electric field (i.e., quadratic in electric field amplitudes $\mathbf{E}$ and $\mathbf{E}^{*}$ ), and linear in $\mathbf{B}_{\|}$. Second order (in electric field) effects generally require breaking of spatial inversion symmetry [28-30], and, here, the coefficient $M_{1}$ changes sign upon $z \rightarrow-z$ inversion.

Equation (1) describes the contribution arising from a perfectly quadratic dispersion relation $\epsilon=p^{2} / 2 m$ ( $p$ is the magnitude of momentum and $m$ is mass) when the relaxation rates are independent of energy, and this contribution will generally dominate in bilayer graphene (there will be small corrections when these conditions are not exactly met, as described in detail in Sec. III). The parameter $M_{1}$ describes the response to incoming linearly polarized light and we find that

$$
M_{1} \approx-\frac{g e^{3} p^{2}}{16 \pi^{2} \hbar^{4}} \frac{\left(2 \Lambda_{1}+\epsilon \Lambda_{1}^{\prime}\right)}{\Upsilon^{0,1} \Upsilon^{0,2}}\left(\frac{1}{\Upsilon^{1,1}}+\frac{1}{\Upsilon^{-1,1}}\right),
$$

where $g$ is a degeneracy factor ( $g=4$ for spin and valley in graphene) and the electronic charge is $-e, e>0$. For a particular material, the parameter $\Lambda_{1}$ (which is independent of $\omega$ and $\omega_{c}$ ) characterizes the strength of scattering in the presence of an in-plane magnetic field and $z \rightarrow-z$ asymmetry (we will present the explicit form of $\Lambda_{1}$ for bilayer graphene later). The functions $\Upsilon^{\ell, j}$, with integer $\ell$ and $j$, where

$$
\Upsilon^{\ell, j}=\tau_{|j|}^{-1}-i \ell \omega+i j \omega_{c}, \quad \tau_{1} \equiv \tau,
$$

account for the dependence of the current on electric-field frequency $\omega$ and cyclotron frequency $\omega_{c}=e B_{\perp} v_{g} / p$ including the cyclotron resonance effect $[6,31,32]$. Here $B_{\perp}=\left|\mathbf{B}_{\perp}\right|$ and we consider $\mathbf{B}_{\perp}=B_{\perp} \hat{\mathbf{n}}_{z}$. Also, $v_{g}$ is the group velocity $v_{g}=d \epsilon / d p$, so $\omega_{c}=e B_{\perp} v^{2} / \epsilon$ [33] for linear dispersion $\epsilon=v p$, and $\omega_{c}=e B_{\perp} / m$ [34] for quadratic dispersion $\epsilon=$ $p^{2} /(2 m)$. Parameters $\tau_{1}$ and $\tau_{2}$ are relaxation times for the first and second angular harmonics of the electronic distribution, 


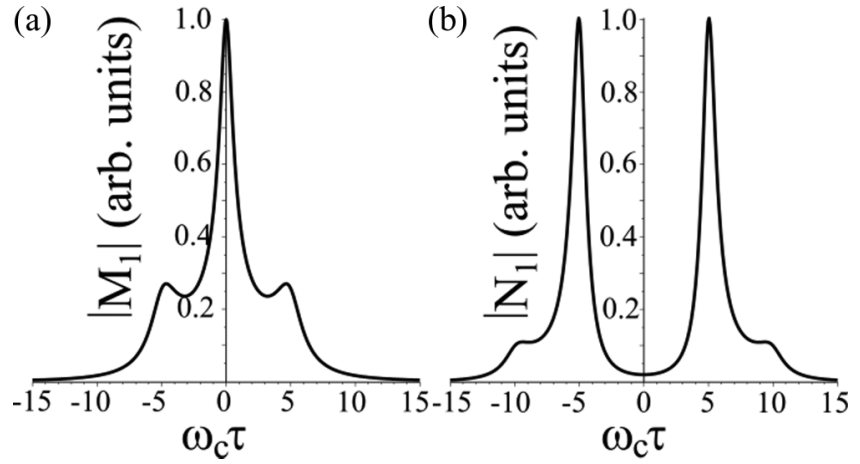

FIG. 1. (a) Magnitude of the nonlinear coefficient $\left|M_{1}\right|$, Eq. (3), describing the magnetic ratchet effect as a function of cyclotron frequency $\omega_{c}$ for fixed $\omega$. (b) Magnitude of the nonlinear coefficient $\left|N_{1}\right|$, Eq. (6), describing second harmonic generation as a function of $\omega_{c}$. For both plots, $\omega \tau=5$ and $\tau_{2}=\tau$.

respectively ( $\tau_{1}$ is the usual momentum relaxation rate that we denote as $\tau$ from now on).

Figure 1(a) shows the magnitude of the nonlinear coefficient $\left|M_{1}\right|$ as a function of cyclotron frequency $\omega_{c}$ for fixed $\omega$. Although there is a noticeable resonance for $\omega_{c}=\omega$ [due to the presence of $\Upsilon^{ \pm 1,1}$ in Eq. (3)], the ratchet effect is actually strongest for $\omega_{c}=0$ because of the product $\Upsilon^{0,1} \Upsilon^{0,2}$. This product arises because of the need to couple with dc components of the electronic distribution in order to create dc current.

The second harmonic current density $\mathbf{J}^{(2)}$ is given by

$$
\begin{aligned}
\mathbf{J}^{(2) \approx} & 2 \operatorname{Re}\left\{\left[2\left[\left(\mathbf{B}_{\|} \times \hat{\mathbf{n}}_{z}\right) \cdot \mathbf{E}\right] \mathbf{E}-\left(\mathbf{B}_{\|} \times \hat{\mathbf{n}}_{z}\right) \mathbf{E}^{2}\right] N_{1} e^{-2 i \omega t}\right\} \\
& -2 \operatorname{Re}\left\{\left[2\left(\mathbf{B}_{\|} \cdot \mathbf{E}\right) \mathbf{E}-\mathbf{B}_{\|} \mathbf{E}^{2}\right] N_{2} e^{-2 i \omega t}\right\},
\end{aligned}
$$

where $\mathbf{E}^{2} \equiv \mathbf{E} \cdot \mathbf{E}=E_{x}^{2}+E_{y}^{2}$. This describes the contribution arising from a perfectly quadratic dispersion relation when the relaxation rates are independent of energy (there will be small corrections when these conditions are not exactly met, as described in detail in Sec. III). These terms describe an effect similar to the Faraday effect [28] in that incoming planepolarized light results in the emission of a plane-polarized second harmonic with the magnetic field contributing to a rotation of the angle of polarization. Incoming circularly polarized light results in circularly polarized second harmonic generation. The coefficients $N_{1}, N_{2}$ are given by

$$
\begin{aligned}
N_{1}= & -\frac{g e^{3} p^{2}}{32 \pi^{2} \hbar^{4}}\left(\Lambda_{1}+\epsilon \Lambda_{1}^{\prime}\right) \\
& \times\left(\frac{1}{\Upsilon^{1,1} \Upsilon^{2,2} \Upsilon^{2,1}}+\frac{1}{\Upsilon^{1,-1} \Upsilon^{2,-2} \Upsilon^{2,-1}}\right), \\
N_{2}= & -\frac{i g e^{3} p^{2}}{32 \pi^{2} \hbar^{4}}\left(\Lambda_{1}+\epsilon \Lambda_{1}^{\prime}\right) \\
& \times\left(\frac{1}{\Upsilon^{1,1} \Upsilon^{2,2} \Upsilon^{2,1}}-\frac{1}{\Upsilon^{1,-1} \Upsilon^{2,-2} \Upsilon^{2,-1}}\right)
\end{aligned}
$$

Figure 1(b) shows the magnitude of the nonlinear coefficient $\left|N_{1}\right|$ as a function of cyclotron frequency $\omega_{c}$ for fixed $\omega$ (note that $\left|N_{2}\right|$ shows almost the same dependence on $\omega_{c}$, both qualitatively and quantitatively, except that $\left|N_{2}\right|=0$ for $\left.\omega_{c}=0\right)$. In stark contrast to $\left|M_{1}\right|$, the resonance at $\omega_{c}=\omega$ is far stronger than the signal at $\omega_{c}=0$ because there are no dc components of the electronic distribution involved (i.e., no $\Upsilon^{0, j}$ factors), and there is also a resonance at $\omega_{c}=2 \omega$.

The combinations of electric and magnetic fields in Eqs. (1) and (5) satisfy spatial symmetries including rotations in the two-dimensional plane $(x-y)$ of the sample. As $\mathbf{B}_{\|}$is an axial vector, the combination $\mathbf{B}_{\|} \times \hat{\mathbf{n}}_{z}$, appearing in the $\operatorname{Re}\left(M_{1}\right)$ and $N_{1}$ terms, behaves as a true vector. However, factors in the $\operatorname{Im}\left(M_{1}\right)$ and $N_{2}$ terms containing $\mathbf{B}_{\|}$appear to break some reflection symmetries (e.g., reflection in a plane perpendicular to the sample, such as the $y-z$ plane) but they are in fact satisfied because $\operatorname{Im}\left(M_{1}\right)$ and $N_{2}$ are odd functions of the combination $\hat{\mathbf{n}}_{z} \cdot \mathbf{B}_{\perp}$ and, thus, change sign upon such reflections. Hence, $\operatorname{Im}\left(M_{1}\right)=0$ and $N_{2}=0$ when $\mathbf{B}_{\perp}=0$ whereas $\operatorname{Re}\left(M_{1}\right)$ and $N_{1}$ are even functions of $\hat{\mathbf{n}}_{z} \cdot \mathbf{B}_{\perp}$ and are generally nonzero for $\mathbf{B}_{\perp}=0$.

In the next section, we describe a phenomenological Drude model in which the ratchet effect may be viewed as arising from the motion of classical particles in the presence of friction created by the in-plane magnetic field. This model correctly accounts for the combinations of fields in Eqs. (1) and (5), but does not quite account for the frequency dependencies in Eqs. (3), (6), and (7) because it only contains one relaxation rate $\tau$. However, in Sec. III, we use the Boltzmann equation to derive general equations for the dc current and second harmonic for a magnetic ratchet in an arbitrary two-dimensional electron system with $z \rightarrow-z$ asymmetry and an isotropic dispersion $\epsilon(p)$, including cyclotron motion, too. Section IV describes scattering in the presence of an in-plane magnetic field in bilayer graphene in order to apply the general equations to it, resulting in the simplified expressions Eqs. (1) and (5). In Sec. V, we show that, for $\mathbf{B}_{\|}=0$, trigonal warping in bilayer graphene leads to a valley current of large magnitude which, when summed over both valleys, yields zero total current, unless there is an additional source of valley polarization.

\section{PHENOMENOLOGICAL DRUDE MODEL}

The expressions (1) and (5) for nonlinear current densities may be viewed as being due to the motion of classical particles in the presence of friction created by the in-plane magnetic field. Previously [1,3], a classical Drude model has been used to provide a simple picture of the origin of ratchet current for intraband transitions. Here, we generalize this approach for the case where the nonlinearity is produced by the inplane magnetic field, and we consider cyclotron motion and second harmonic generation, too. We consider the equation of motion for the average drift velocity $\mathbf{v}_{d}$ per electron in a two-dimensional system:

$$
m \frac{d \mathbf{v}_{d}}{d t}=-e \mathbf{E}-e\left(\mathbf{v}_{d} \times \mathbf{B}_{\perp}\right)-\frac{m \mathbf{v}_{d}}{\tau}+\mathbf{F}\left(\mathbf{v}_{d}, \mathbf{B}_{\|}\right),
$$

where $\tau$ is the momentum relaxation time, and the perpendicular magnetic field $\mathbf{B}_{\perp}$ and in-plane ac electric field $\mathbf{E}(t)$, Eq. (2), enter via the Lorentz force. The term $\mathbf{F}\left(\mathbf{v}_{d}, \mathbf{B}_{\|}\right)$describes friction due to the presence of the in-plane magnetic field $\mathbf{B}_{\|}$ which introduces nonlinearity into the system. We assume this term is quadratic in velocity $\mathbf{v}_{d}$ and linear in magnetic field $\mathbf{B}_{\|}$:

$$
\mathbf{F}\left(\mathbf{v}_{d}, \mathbf{B}_{\|}\right)=\alpha\left\{2\left[\left(\mathbf{B}_{\|} \times \hat{\mathbf{n}}_{z}\right) \cdot \mathbf{v}_{d}\right] \mathbf{v}_{d}-\left(\mathbf{B}_{\|} \times \hat{\mathbf{n}}_{z}\right)\left|\mathbf{v}_{d}\right|^{2}\right\},
$$


where $\alpha$ is a phenomenological parameter that characterizes the material properties of the particular system in question. This form of the friction term is obtained by requiring that it behaves as a true vector rather than, say, an axial vector (as $\mathbf{B}_{\|}$ is an axial vector, the combination $\mathbf{B}_{\|} \times \hat{\mathbf{n}}_{z}$ behaves as a true vector). In principle, there should be a second phenomenological parameter to characterize the relative weight of the two terms in $\mathbf{F}\left(\mathbf{v}_{d}, \mathbf{B}_{\|}\right)$, but, for simplicity, we insert its value above [it is determined by demanding that we obtain nonlinear contributions with the correct combinations of fields Eqs. (1) and (5)].

To solve Eq. (8) we use a harmonic expansion of the velocity,

$$
\mathbf{v}_{d}(t)=\sum_{\ell=0, \pm 1, \ldots} \mathbf{v}_{d}^{(\ell)} e^{-i n \omega t},
$$

which yields coupled equations for the coefficients $\mathbf{v}_{d}^{(\ell)}$ :

$$
\begin{aligned}
-i \ell \omega \mathbf{v}_{d}^{(\ell)}= & -\frac{e \mathbf{E}}{m} \delta_{\ell, 1}-\frac{e \mathbf{E}^{*}}{m} \delta_{\ell,-1}-\omega_{c}\left(\mathbf{v}_{d}^{(\ell)} \times \hat{\mathbf{n}}_{z}\right)-\frac{\mathbf{v}_{d}^{(\ell)}}{\tau} \\
& +\frac{\alpha}{m} \sum_{n}\left\{2\left[\left(\mathbf{B}_{\|} \times \hat{\mathbf{n}}_{z}\right) \cdot \mathbf{v}_{d}^{(n)}\right] \mathbf{v}_{d}^{(\ell-n)}\right. \\
& \left.-\left(\mathbf{B}_{\|} \times \hat{\mathbf{n}}_{z}\right)\left[\mathbf{v}_{d}^{(n)} \cdot \mathbf{v}_{d}^{(\ell-n)}\right]\right\}
\end{aligned}
$$

Neglecting the in-plane magnetic field, the linear harmonics are given by

$$
\begin{aligned}
& \left(\mathbf{v}_{d}^{(1)}\right)_{x}=-\frac{e \tau}{m}\left[\frac{(1-i \omega \tau) E_{x}-\omega_{c} \tau E_{y}}{(1-i \omega \tau)^{2}+\left(\omega_{c} \tau\right)^{2}}\right], \\
& \left(\mathbf{v}_{d}^{(1)}\right)_{y}=-\frac{e \tau}{m}\left[\frac{(1-i \omega \tau) E_{y}+\omega_{c} \tau E_{x}}{(1-i \omega \tau)^{2}+\left(\omega_{c} \tau\right)^{2}}\right],
\end{aligned}
$$

and $\mathbf{v}_{d}^{(-1)}=\left(\mathbf{v}_{d}^{(1)}\right)^{*}$. Since current density is related to drift velocity by $\mathbf{J}(t)=-n e \mathbf{v}_{d}(t)$ where $n$ is the electron number density [34], the linear current density may be written as $\mathbf{J}^{(1)}=-2 n e \operatorname{Re}\left\{\mathbf{v}_{d}^{(1)} e^{-i \omega t}\right\}$ which yields $\mathbf{J}^{(1)}=2 \operatorname{Re}\left\{\sigma \mathbf{E} e^{-i \omega t}\right\}$ where the conductivity tensor $\sigma$ has components

$$
\begin{gathered}
\sigma_{x x}=\sigma_{y y}=\frac{(1-i \omega \tau) \sigma_{0}}{(1-i \omega \tau)^{2}+\left(\omega_{c} \tau\right)^{2}}, \\
\sigma_{x y}=-\sigma_{y x}=-\frac{\omega_{c} \tau \sigma_{0}}{(1-i \omega \tau)^{2}+\left(\omega_{c} \tau\right)^{2}},
\end{gathered}
$$

with dc Drude conductivity $\sigma_{0}=n e^{2} \tau / m$, as expected [34].

The in-plane magnetic field introduces nonlinearity which couples nonlinear harmonics to the linear one, Eq. (9). To first order in $\mathbf{B}_{\|}$, we find that the ratchet current density is given by Eq. (1) and the Drude expression for the nonlinear coefficient $M_{1}$ is

$$
M_{1}^{D}=-\frac{\alpha n e^{3}}{m^{3}} \frac{1}{\left(\Upsilon^{0,1}\right)^{2}}\left(\frac{1}{\Upsilon^{1,1}}+\frac{1}{\Upsilon^{-1,1}}\right)
$$

Likewise, the second harmonic current density is given by Eq. (5) and the Drude expressions for the nonlinear coefficients
$N_{1}, N_{2}$ are given by

$$
\begin{aligned}
& N_{1}^{D}=-\frac{\alpha n e^{3}}{2 m^{3}}\left[\frac{1}{\left(\Upsilon^{1,1}\right)^{2} \Upsilon^{2,1}}+\frac{1}{\left(\Upsilon^{1,-1}\right)^{2} \Upsilon^{2,-1}}\right], \\
& N_{2}^{D}=-\frac{i \alpha n e^{3}}{2 m^{3}}\left[\frac{1}{\left(\Upsilon^{1,1}\right)^{2} \Upsilon^{2,1}}-\frac{1}{\left(\Upsilon^{1,-1}\right)^{2} \Upsilon^{2,-1}}\right] .
\end{aligned}
$$

Comparison with the coefficients derived using the Boltzmann equations (3), (6), and (7) shows that although the simple Drude model correctly produces the correct combinations of fields Eqs. (1) and (5), it does not quite account for the frequency dependence because it only contains one relaxation rate $\tau$ [the parameter $\tau_{2}$ describing relaxation of the second angular harmonic of the electronic distribution is not included in Eq. (8)]. In order to accurately describe temporal and spatial relaxation of the electronic distribution it is necessary to employ the Boltzmann equation, as described in the next section.

\section{BOLTZMANN EQUATION}

In this section, we derive the intraband contribution to the second order (in electric field) nonlinear conductivity due to the presence of an in-plane magnetic field $\mathbf{B}_{\|}$for an arbitrary two-dimensional electron system with $z \rightarrow-z$ asymmetry and an isotropic dispersion $\epsilon(p)$. We consider linear-in- $\mathbf{B}_{\|}$terms and we take into account the effect of a perpendicular magnetic field $\mathbf{B}_{\perp}$ which introduces cyclotron motion with cyclotron frequency $\omega_{c}=e B_{\perp} v_{g} / p$. These semiclassical calculations are valid for finite Fermi energy $\epsilon_{F}$ with $\epsilon_{F} \gg\{\hbar / \tau, \hbar \omega\}$, $\omega_{c} \tau \ll 1$, and we also assume that the electrons are degenerate $\epsilon_{F} \gg k_{B} T$.

We consider a spatially homogeneous system with electron motion described by the Boltzmann equation [34],

$$
-e\left(\mathbf{E}_{\|}+\mathbf{v}_{g} \times \mathbf{B}_{\perp}\right) \cdot \nabla_{p} f(\mathbf{p}, t)+\frac{\partial f(\mathbf{p}, t)}{\partial t}=S\{f\},
$$

where the electron distribution $f(\mathbf{p}, t)$ is a function of momentum $\mathbf{p}$ and time $t, \mathbf{v}_{g}=v_{g}(\hat{\mathbf{i}} \cos \phi+\hat{\boldsymbol{j}} \sin \phi)$ and $\phi$ is the polar angle of momentum. The in-plane alternating field $\mathbf{E}_{\|}(t)$, Eq. (2), and the perpendicular magnetic field $\mathbf{B}_{\perp}=B_{\perp} \hat{\mathbf{n}}_{z}$ are accounted for by the Lorentz force, while the in-plane magnetic field $\mathbf{B}_{\|}$enters via the the collision integral $S\{f\}$,

$$
S\{f\}=\sum_{\mathbf{p}^{\prime}}\left[W_{\mathbf{p p}^{\prime}} f\left(\mathbf{p}^{\prime}, t\right)-W_{\mathbf{p}^{\prime} \mathbf{p}} f(\mathbf{p}, t)\right] .
$$

Here $W_{\mathbf{p}^{\prime} \mathbf{p}}$ is the scattering rate describing scattering from initial $|\mathbf{p}\rangle$ to final state $\left|\mathbf{p}^{\prime}\right\rangle$ in the presence of a scattering potential $\delta H$, as given by Fermi's "golden rule,"

$$
W_{\mathbf{p}^{\prime} \mathbf{p}}=\frac{2 \pi}{\hbar}\left|\left\langle\mathbf{p}^{\prime}|\delta H| \mathbf{p}\right\rangle\right|^{2} \delta\left(\epsilon_{\mathbf{p}}-\epsilon_{\mathbf{p}^{\prime}}\right) .
$$

We consider static impurities

$$
\delta H=\sum_{j=1}^{N_{i}} \hat{Y} u\left(\mathbf{r}-\mathbf{R}_{j}\right),
$$

where $N_{i}$ is the number of impurities, $u\left(\mathbf{r}-\mathbf{R}_{j}\right)$ describes the spatial dependence of the impurity potential. The dimensionless matrix $\hat{Y}$ takes account of any additional degree of freedom related to structure within the unit cell, for example $A / B$ 
lattice in graphene. We neglect interference between different impurities and use the Fourier transform of the impurity potential,

$$
\tilde{u}(\mathbf{q})=\int d^{2} r u(\mathbf{r}) e^{-i \mathbf{q} \cdot \mathbf{r} / \hbar} .
$$

When evaluating the scattering rate (14), we expand the states $|\mathbf{p}\rangle,\left|\mathbf{p}^{\prime}\right\rangle$ in powers of the in-plane magnetic field $\mathbf{B}_{\|}$. For zeroth order, $\mathbf{B}_{\|}=0$, we recover the usual relaxation rates for the $j$ th angular harmonics of the electronic distribution,

$$
\tau_{|j|}^{-1}=\frac{2 \pi}{\hbar} \sum_{\mathbf{p}^{\prime}}\left|\left\langle\mathbf{p}^{\prime}|\delta H| \mathbf{p}\right\rangle\right|^{2} \delta\left(\epsilon_{\mathbf{p}}-\epsilon_{\mathbf{p}^{\prime}}\right)\left[1-\cos \left(j\left[\phi^{\prime}-\phi\right]\right)\right],
$$

and we write $\tau_{1} \equiv \tau$. To linear order in $\mathbf{B}_{\|}$, we find that the scattering rate may be written generically as

$$
\begin{aligned}
\delta W_{\mathbf{p}^{\prime} \mathbf{p}}= & \frac{1}{L^{2}}\left|\tilde{u}\left(\mathbf{p}^{\prime}-\mathbf{p}\right)\right|^{2} \delta\left(\epsilon_{\mathbf{p}}-\epsilon_{\mathbf{p}^{\prime}}\right) \\
& \times\left\{\{ \Omega - \Omega _ { c } \operatorname { c o s } [ 2 ( \phi ^ { \prime } - \phi ) ] \} \left[B_{x}\left(p_{y}^{\prime}+p_{y}\right)\right.\right. \\
& \left.-B_{y}\left(p_{x}^{\prime}+p_{x}\right)\right]+\Omega_{s} \sin \left[2\left(\phi^{\prime}-\phi\right)\right]\left[B_{x}\left(p_{x}^{\prime}-p_{x}\right)\right. \\
& \left.\left.+B_{y}\left(p_{y}^{\prime}-p_{y}\right)\right]\right\},
\end{aligned}
$$

where $\Omega, \Omega_{c}, \Omega_{s}$ are angle-independent factors that depend on specific material properties.

In order to solve the Boltzmann equation (12), we use polar coordinates $(p, \phi)$ for momentum and expand the distribution function in terms of $\phi$ and $t$ harmonics with coefficients $f_{m}^{(n)}$ :

$$
f(\mathbf{p}, t)=\sum_{n, m} f_{m}^{(n)} e^{i m \phi-i n \omega t},
$$

where $m, n$ are integers. We also perform a harmonic expansion of the impurity potential,

$$
\left|\tilde{u}\left(\mathbf{p}^{\prime}-\mathbf{p}\right)\right|^{2}=\sum_{m} v_{m} e^{i m\left(\phi^{\prime}-\phi\right)},
$$

with the constraint that $v_{-m}=v_{m}$ as it is an even function of $\left(\phi^{\prime}-\phi\right)$. Then, we multiply the Boltzmann equation by a factor $\exp (-i j \phi+i \ell \omega t)$, where $j, \ell$ are integers, and integrate over a period $2 \pi$ of angle $\phi$ and a period of time $t$. This results in coupling between different harmonic coefficients:

$$
\begin{aligned}
f_{j}^{(\ell)}\left(\tau_{|j|}^{-1}-i \ell \omega+i j \omega_{c}\right)= & \alpha_{j-1} f_{j-1}^{(\ell-1)}+\eta_{j+1} f_{j+1}^{(\ell-1)} \\
& +\tilde{\alpha}_{j-1} f_{j-1}^{(\ell+1)}+\tilde{\eta}_{j+1} f_{j+1}^{(\ell+1)}+\delta S_{j}^{(\ell)} .
\end{aligned}
$$

Operators $\alpha, \eta$ are linear in the electric field,

$$
\begin{aligned}
\alpha_{j} & =\frac{e\left(E_{x}-i E_{y}\right)}{2}\left(-\frac{j}{p}+\frac{\partial}{\partial p}\right), \\
\tilde{\alpha}_{j} & =\frac{e\left(E_{x}^{*}-i E_{y}^{*}\right)}{2}\left(-\frac{j}{p}+\frac{\partial}{\partial p}\right), \\
\eta_{j} & =\frac{e\left(E_{x}+i E_{y}\right)}{2}\left(\frac{j}{p}+\frac{\partial}{\partial p}\right), \\
\tilde{\eta}_{j} & =\frac{e\left(E_{x}^{*}+i E_{y}^{*}\right)}{2}\left(\frac{j}{p}+\frac{\partial}{\partial p}\right),
\end{aligned}
$$

and factors $\delta S_{j}^{(\ell)}$ account for the linear-in- $\mathbf{B}_{\|}$correction to scattering; the relevant ones have small values of $j$ :

$$
\begin{aligned}
\delta S_{0}^{(\ell)} & =0, \\
\delta S_{1}^{(\ell)} & =\frac{1}{2} p \Gamma(\epsilon)\left(B_{y}-i B_{x}\right) \Lambda_{1} f_{2}^{(\ell)}, \\
\delta S_{-1}^{(\ell)} & =\frac{1}{2} p \Gamma(\epsilon)\left(B_{y}+i B_{x}\right) \Lambda_{1} f_{-2}^{(\ell)}, \\
\delta S_{2}^{(\ell)} & =\frac{1}{2} p \Gamma(\epsilon)\left[\left(B_{y}+i B_{x}\right) \Lambda_{1} f_{1}^{(\ell)}+\left(B_{y}-i B_{x}\right) \Lambda_{2} f_{3}^{(\ell)}\right], \\
\delta S_{-2}^{(\ell)} & =\frac{1}{2} p \Gamma(\epsilon)\left[\left(B_{y}-i B_{x}\right) \Lambda_{1} f_{-1}^{(\ell)}+\left(B_{y}+i B_{x}\right) \Lambda_{2} f_{-3}^{(\ell)}\right] .
\end{aligned}
$$

Here, $\Gamma(\epsilon)$ is the electronic density of states per spin and per valley, per unit area $\Gamma=p /\left(2 \pi \hbar^{2} v_{g}\right)$; the parameters $\Lambda_{1}, \Lambda_{2}$ are given by

$$
\begin{gathered}
\Lambda_{1}=\Omega\left(v_{0}-v_{2}\right)+\frac{1}{2} \Omega_{c}\left(v_{0}-2 v_{2}+v_{4}\right) \\
+\frac{1}{2} \Omega_{s}\left(v_{0}-2 v_{1}+2 v_{3}-v_{4}\right), \\
\Lambda_{2}=\Omega\left(v_{0}+v_{1}-v_{2}-v_{3}\right)+\frac{1}{2} \Omega_{c}\left(v_{0}-2 v_{2}-v_{3}+v_{4}+v_{5}\right) \\
-\frac{1}{2} \Omega_{s}\left(v_{0}-v_{3}-v_{4}+v_{5}\right) .
\end{gathered}
$$

The current density is given by [34]

$$
\mathbf{J}=-\frac{g e}{L^{2}} \sum_{\mathbf{p}} \mathbf{v}_{g} f(\mathbf{p}, t),
$$

where $g$ is a degeneracy factor ( $g=4$ for spin and valley in graphene). Owing to the angular factors in $\mathbf{v}_{g}$, only the firstorder angular harmonics $(m= \pm 1)$ in the harmonic expansion (17) survive after integrating over all angles $\phi$. We express the current as a series in temporal harmonics as

$$
\begin{aligned}
\mathbf{J}= & \mathbf{J}^{(0)}+\mathbf{J}^{(1)}+\mathbf{J}^{(2)}+\cdots, \\
\mathbf{J}^{(0)}= & -\frac{g e}{L^{2}} \sum_{\mathbf{p}} \mathbf{v}_{g}\left(f_{1}^{(0)} e^{i \phi}+f_{-1}^{(0)} e^{-i \phi}\right), \\
\mathbf{J}^{(n)}= & -\frac{g e}{L^{2}} \sum_{\mathbf{p}} \mathbf{v}_{g}\left[\left(f_{1}^{(n)} e^{i \phi}+f_{-1}^{(n)} e^{-i \phi}\right) e^{-i n \omega t}\right. \\
& \left.+\left(f_{1}^{(-n)} e^{i \phi}+f_{-1}^{(-n)} e^{-i \phi}\right) e^{i n \omega t}\right] ; \quad n \geqslant 1 .
\end{aligned}
$$

The coupled equations (18) are used to express harmonics $f_{j}^{(\ell)}$ in terms of the equilibrium distribution $f_{0}^{(0)}$. Thus, it is possible to calculate each of the harmonic current densities $\mathbf{J}^{(0)}, \mathbf{J}^{(1)}, \mathbf{J}^{(2)}$, which we describe below, beginning with the linear response $\mathbf{J}^{(1)}$.

\section{A. Linear response $J^{(1)}$}

The leading contribution to the linear current density $J^{(1)}$ arises from the linear-in-electric-field terms in (18) (i.e., $\delta S_{j}^{(\ell)}$ is irrelevant) with

$$
f_{ \pm 1}^{(1)}=\frac{e \tau\left(E_{x} \mp i E_{y}\right)}{2\left(1-i \omega \tau \pm i \omega_{c} \tau\right)} \frac{\partial f_{0}^{(0)}}{\partial p} ; \quad f_{ \pm 1}^{(-1)}=\left(f_{\mp 1}^{(1)}\right)^{*} .
$$

For a degenerate electron gas, $\epsilon_{F} \gg k_{B} T$, we find that $\mathbf{J}^{(1)}=$ $2 \operatorname{Re}\left\{\sigma \mathbf{E} e^{-i \omega t}\right\}$ where the conductivity tensor $\sigma$ has components as given in Eqs. (10) and (11) with dc Drude conductivity $\sigma_{0}=g e^{2} v_{g} p \tau /\left(4 \pi \hbar^{2}\right)$ (all parameters are evaluated on the Fermi surface). For a system with quadratic dispersion $\epsilon=$ $p^{2} /(2 m)$ (such as bilayer graphene), then $\sigma_{0}=g e^{2} \epsilon \tau /\left(2 \pi \hbar^{2}\right)$ 
[35], for linear dispersion $\epsilon=v p$ (monolayer graphene), then $\sigma_{0}=g e^{2} \epsilon \tau /\left(4 \pi \hbar^{2}\right)[33,36,37]$.

\section{B. Ratchet de current $\mathbf{J}^{(0)}$}

The dc current density, Eq. (21), may be written as

$$
\begin{aligned}
\mathbf{J}^{(0)}= & \left\{\left[\left(\mathbf{B}_{\|} \times \hat{\mathbf{n}}_{z}\right) \cdot \mathbf{E}\right] \mathbf{E}^{*}+\left[\left(\mathbf{B}_{\|} \times \hat{\mathbf{n}}_{z}\right) \cdot \mathbf{E}^{*}\right] \mathbf{E}\right. \\
& \left.-\left(\mathbf{B}_{\|} \times \hat{\mathbf{n}}_{z}\right)|\mathbf{E}|^{2}\right\} \operatorname{Re}\left(M_{1}\right)+\left(\mathbf{B}_{\|} \times \hat{\mathbf{n}}_{z}\right)|\mathbf{E}|^{2} \operatorname{Re}\left(M_{2}\right) \\
& +i \mathbf{B}_{\|}\left[\left(\mathbf{E} \times \mathbf{E}^{*}\right) \cdot \hat{\mathbf{n}}_{z}\right] \operatorname{Re}\left(M_{3}\right)+\left\{\mathbf{E}\left(\mathbf{B}_{\|} \cdot \mathbf{E}^{*}\right)\right. \\
& \left.+\mathbf{E}^{*}\left(\mathbf{B}_{\|} \cdot \mathbf{E}\right)-\mathbf{B}_{\|}|\mathbf{E}|^{2}\right\} \operatorname{Im}\left(M_{1}\right)-\mathbf{B}_{\|}|\mathbf{E}|^{2} \operatorname{Im}\left(M_{2}\right) \\
& +i \mathbf{B}_{\|} \times\left(\mathbf{E} \times \mathbf{E}^{*}\right) \operatorname{Im}\left(M_{3}\right) .
\end{aligned}
$$

In terms of components, this may be expressed [6] as

$$
\begin{aligned}
J_{x}^{(0)}= & B_{x}\left(-|E|^{2} \operatorname{Im} M_{2}+\Theta_{1} \operatorname{Im} M_{1}-\Theta_{2} \operatorname{Re} M_{1}+\Theta_{3} \operatorname{Re} M_{3}\right) \\
& +B_{y}\left(|E|^{2} \operatorname{Re} M_{2}+\Theta_{1} \operatorname{Re} M_{1}+\Theta_{2} \operatorname{Im} M_{1}+\Theta_{3} \operatorname{Im} M_{3}\right), \\
J_{y}^{(0)}= & B_{x}\left(-|E|^{2} \operatorname{Re} M_{2}+\Theta_{1} \operatorname{Re} M_{1}+\Theta_{2} \operatorname{Im} M_{1}-\Theta_{3} \operatorname{Im} M_{3}\right) \\
& +B_{y}\left(-|E|^{2} \operatorname{Im} M_{2}-\Theta_{1} \operatorname{Im} M_{1}+\Theta_{2} \operatorname{Re} M_{1}+\Theta_{3} \operatorname{Re} M_{3}\right),
\end{aligned}
$$

where $\Theta_{1}=\left(\left|E_{x}\right|^{2}-\left|E_{y}\right|^{2}\right), \Theta_{2}=\left(E_{x} E_{y}^{*}+E_{y} E_{x}^{*}\right)$, and $\Theta_{3}=i\left(E_{x} E_{y}^{*}-E_{y} E_{x}^{*}\right)[38]$.

For a degenerate electron gas, $\epsilon_{F} \gg k_{B} T$, we find the three coefficients are given by

$$
\begin{aligned}
M_{1}= & -\frac{g e^{3}}{32 \pi^{2} \hbar^{4}}\left(\frac{1}{\Upsilon^{1,1}}+\frac{1}{\Upsilon^{-1,1}}\right) \\
& \times\left[\frac{4 \Lambda_{1} p^{2}}{\Upsilon^{0,1} \Upsilon^{0,2}}+v_{g} p^{3}\left(\frac{\Lambda_{1}}{\Upsilon^{0,1} \Upsilon^{0,2}}\right)^{\prime}\right], \\
M_{2}= & \frac{g e^{3} \Lambda_{1} p^{2}}{32 \pi^{2} \hbar^{4}}\left(\frac{1}{\Upsilon^{1,2} \Upsilon^{1,1}}+\frac{1}{\Upsilon^{-1,2} \Upsilon^{-1,1}}\right) \\
& \times\left[\frac{1}{\Upsilon^{0,1}}-\frac{p v_{g}^{\prime}}{\Upsilon^{0,1}}-v_{g} p\left(\frac{1}{\Upsilon^{0,1}}\right)^{\prime}\right], \\
M_{3}= & \frac{i g e^{3} \Lambda_{1} p^{2}}{32 \pi^{2} \hbar^{4}}\left(\frac{1}{\Upsilon^{1,2} \Upsilon^{1,1}}-\frac{1}{\Upsilon^{-1,2} \Upsilon^{-1,1}}\right) \\
& \times\left[\frac{1}{\Upsilon^{0,1}}-\frac{p v_{g}^{\prime}}{\Upsilon^{0,1}}-v_{g} p\left(\frac{1}{\Upsilon^{0,1}}\right)^{\prime}\right],
\end{aligned}
$$

where $(\ldots)^{\prime} \equiv \partial(\ldots) / \partial \epsilon$ and all parameters are evaluated on the Fermi surface. The terms $\operatorname{Re}\left(M_{1}\right), \operatorname{Re}\left(M_{2}\right), \operatorname{Re}\left(M_{3}\right)$ are all even functions of $\hat{\mathbf{n}}_{z} \cdot \mathbf{B}_{\perp}$, whereas $\operatorname{Im}\left(M_{1}\right), \operatorname{Im}\left(M_{2}\right)$, $\operatorname{Im}\left(M_{3}\right)$ are odd functions, thus they are zero for $\mathbf{B}_{\perp}=0$. These equations generalize those in Refs. $[3,6,8,10]$ and describe the intraband contribution to the ratchet effect in a twodimensional material with isotropic dispersion. Parameters such as the scattering times $\tau, \tau_{2}$, Eq. (15), and $\Lambda_{1}$, Eq. (19), are specific to the given material; we will describe them for bilayer graphene in Sec. IV.

The coefficients $M_{1}, M_{2}, M_{3}$ describe the response to different polarizations of light: $M_{2}$ characterizes the effect of unpolarized light, $M_{1}$ describes additional terms that appear if the light is linearly polarized, $M_{3}$ includes additional terms that occur for circular polarization. In particular, for incoming linearly polarized light, $E_{x}(t)=E_{0} \cos \theta \cos \omega t$ and $E_{y}(t)=$
$E_{0} \sin \theta \cos \omega t$ where $\theta$ is the polarization angle, then

$$
E_{x}^{*}=E_{x}=\frac{E_{0}}{2} \cos \theta ; \quad E_{y}^{*}=E_{y}=\frac{E_{0}}{2} \sin \theta .
$$

In this case, the current density may be expressed as

$$
\begin{aligned}
\mathbf{J}^{(0)}= & \frac{E_{0}^{2}}{4} B_{\|}\left|M_{1}\right|\left\{\hat{\mathbf{i}} \cos \left(2 \theta-\varphi-\chi_{1}+\pi / 2\right)\right. \\
& \left.+\hat{\boldsymbol{\jmath}} \sin \left(2 \theta-\varphi-\chi_{1}+\pi / 2\right)\right\}, \\
& +\frac{E_{0}^{2}}{4}\left\{\left(\mathbf{B}_{\|} \times \hat{\mathbf{n}}_{z}\right) \operatorname{Re} M_{2}-\mathbf{B}_{\|} \operatorname{Im} M_{2}\right\},
\end{aligned}
$$

where $\chi_{1}=\arg \left(M_{1}\right)$ and $\varphi$ is the polar angle of the in-plane magnetic field $\mathbf{B}_{\|}=\left(B_{x}, B_{y}, 0\right)=B_{\|}(\cos \varphi, \sin \varphi, 0)$ and $B_{\|}=$ $\left|\mathbf{B}_{\|}\right|$. The $M_{1}$ term produces current in a direction determined by the polarization angle $\theta$, the magnetic field direction $\varphi$, and the phase $\chi_{1}$ of the $M_{1}$ coefficient, whereas $M_{2}$ describes current in a direction solely determined by the parallel field. For unpolarized light, the $M_{1}$-related current is zero, but the $M_{2}$ current survives.

For circularly polarized light $E_{x}(t)=E_{0} \cos \omega t$ and $E_{y}(t)=\mu E_{0} \sin \omega t$, where $\mu= \pm 1$ indicates left- or righthanded polarization, then

$$
E_{x}^{*}=E_{x}=\frac{E_{0}}{2} ; \quad E_{y}^{*}=-E_{y}=-i \mu \frac{E_{0}}{2} .
$$

Then, the dc current density is given by

$$
\begin{aligned}
\mathbf{J}^{(0)}= & \frac{E_{0}^{2}}{2} B_{\|}\left|M_{2}\right|\left\{\hat{\mathbf{i}} \cos \left(\varphi-\chi_{2}-\pi / 2\right)\right. \\
& \left.+\hat{\jmath} \sin \left(\varphi-\chi_{2}-\pi / 2\right)\right\}, \\
& +\mu \frac{E_{0}^{2}}{2} B_{\|}\left|M_{3}\right|\left\{\hat{\mathbf{I}} \cos \left(\varphi-\chi_{3}\right)\right. \\
& \left.+\hat{\jmath} \sin \left(\varphi-\chi_{3}\right)\right\},
\end{aligned}
$$

where $\chi_{2}=\arg \left(M_{2}\right), \chi_{3}=\arg \left(M_{3}\right)$, indicating that the direction of the current is determined by the magnetic field direction and the phase of the $M_{2}, M_{3}$ coefficients.

\section{Second harmonic generation $\mathbf{J}^{(2)}$}

The second harmonic of the current density, Eq. (21), may be expressed as

$$
\begin{aligned}
\mathbf{J}^{(2)}= & 2 \operatorname{Re}\left\{\left[\left(\mathbf{B}_{\|} \times \hat{\mathbf{n}}_{z}\right) \mathbf{E}^{2} N_{3}+\mathbf{B}_{\|} \mathbf{E}^{2} N_{4}\right] e^{-2 i \omega t}\right\} \\
& +2 \operatorname{Re}\left\{\left[2\left[\left(\mathbf{B}_{\|} \times \hat{\mathbf{n}}_{z}\right) \cdot \mathbf{E}\right] \mathbf{E}-\left(\mathbf{B}_{\|} \times \hat{\mathbf{n}}_{z}\right) \mathbf{E}^{2}\right] N_{1} e^{-2 i \omega t}\right\} \\
& -2 \operatorname{Re}\left\{\left[2\left(\mathbf{B}_{\|} \cdot \mathbf{E}\right) \mathbf{E}-\mathbf{B}_{\|} \mathbf{E}^{2}\right] N_{2} e^{-2 i \omega t}\right\},
\end{aligned}
$$

where $\mathbf{E}^{2} \equiv \mathbf{E} \cdot \mathbf{E}=E_{x}^{2}+E_{y}^{2}$. In terms of components, this may be written as

$$
\begin{aligned}
J_{x}^{(2)}= & 2 \operatorname{Re}\left\{\left[N_{1}\left(B_{y} \Theta_{4}-B_{x} \Theta_{5}\right)-N_{2}\left(B_{x} \Theta_{4}+B_{y} \Theta_{5}\right)\right.\right. \\
& \left.\left.+\Theta_{6}\left(B_{y} N_{3}+B_{x} N_{4}\right)\right] e^{-2 i \omega t}\right\}, \\
J_{y}^{(2)}= & 2 \operatorname{Re}\left\{\left[N_{1}\left(B_{x} \Theta_{4}+B_{y} \Theta_{5}\right)+N_{2}\left(B_{y} \Theta_{4}-B_{x} \Theta_{5}\right)\right.\right. \\
& \left.\left.+\Theta_{6}\left(-B_{x} N_{3}+B_{y} N_{4}\right)\right] e^{-2 i \omega t}\right\},
\end{aligned}
$$

where $\Theta_{4}=\left(E_{x}^{2}-E_{y}^{2}\right), \Theta_{5}=2 E_{x} E_{y}$, and $\Theta_{6}=\left(E_{x}^{2}+E_{y}^{2}\right)$. For a degenerate electron gas, $\epsilon_{F} \gg k_{B} T$, we find the 
coefficients $N_{i}$ are given by

$$
\begin{aligned}
N_{1}= & -\frac{g e^{3} v_{g} p}{32 \pi \hbar^{2}} \\
& \times\left[\frac{1}{\Upsilon^{1,1}}\left(\frac{v_{g} p \Gamma \Lambda_{1}}{\Upsilon^{2,2} \Upsilon^{2,1}}\right)^{\prime}+\frac{1}{\Upsilon^{1,-1}}\left(\frac{v_{g} p \Gamma \Lambda_{1}}{\Upsilon^{2,-2} \Upsilon^{2,-1}}\right)^{\prime}\right], \\
N_{2}= & -\frac{i g e^{3} v_{g} p}{32 \pi \hbar^{2}} \\
& \times\left[\frac{1}{\Upsilon^{1,1}}\left(\frac{v_{g} p \Gamma \Lambda_{1}}{\Upsilon^{2,2} \Upsilon^{2,1}}\right)^{\prime}-\frac{1}{\Upsilon^{1,-1}}\left(\frac{v_{g} p \Gamma \Lambda_{1}}{\Upsilon^{2,-2} \Upsilon^{2,-1}}\right)^{\prime}\right], \\
N_{3}= & \frac{g e^{3} v_{g} p \Gamma \Lambda_{1}}{32 \pi \hbar^{2}}\left[\frac{1}{\Upsilon^{1,1} \Upsilon^{1,2} \Upsilon^{2,1}}+\frac{1}{\Upsilon^{1,-1} \Upsilon^{1,-2} \Upsilon^{2,-1}}\right. \\
& \left.-\frac{p}{\Upsilon^{1,1} \Upsilon^{1,2}}\left(\frac{v_{g}}{\Upsilon^{2,1}}\right)^{\prime}-\frac{p}{\Upsilon^{1,-1} \Upsilon^{1,-2}}\left(\frac{v_{g}}{\Upsilon^{2,-1}}\right)^{\prime}\right], \\
N_{4}= & \frac{i g e^{3} v_{g} p \Gamma \Lambda_{1}}{32 \pi \hbar^{2}}\left[\frac{1}{\Upsilon^{1,1} \Upsilon^{1,2} \Upsilon^{2,1}}-\frac{1}{\Upsilon^{1,-1} \Upsilon^{1,-2} \Upsilon^{2,-1}}\right. \\
& \left.-\frac{p}{\Upsilon^{1,1} \Upsilon^{1,2}}\left(\frac{v_{g}}{\Upsilon^{2,1}}\right)^{\prime}+\frac{p}{\Upsilon^{1,-1} \Upsilon^{1,-2}}\left(\frac{v_{g}}{\Upsilon^{2,-1}}\right)^{\prime}\right] .
\end{aligned}
$$

Note that coefficients $N_{1}, N_{3}$ are even functions of $\hat{\mathbf{n}}_{z} \cdot \mathbf{B}_{\perp}$, whereas $N_{2}, N_{4}$ are odd, thus $N_{2}=N_{4}=0$ for $\mathbf{B}_{\perp}=0$.

For incoming linearly polarized light Eq. (24), the current density is

$$
\begin{aligned}
\mathbf{J}^{(2)}= & \frac{E_{0}^{2}}{2} B_{\|}\left|N_{1}\right| \cos \left(2 \omega t-\psi_{1}\right)\{\hat{\mathbf{i}} \cos (2 \theta-\varphi+\pi / 2) \\
& +\hat{\boldsymbol{j}} \sin (2 \theta-\varphi+\pi / 2)\}, \\
& +\frac{E_{0}^{2}}{2} B_{\|}\left|N_{2}\right| \cos \left(2 \omega t-\psi_{2}\right)\{\hat{\mathbf{i}} \cos (2 \theta-\varphi+\pi) \\
& +\hat{\boldsymbol{j}} \sin (2 \theta-\varphi+\pi)\}, \\
& +\frac{E_{0}^{2}}{2}\left|N_{3}\right|\left(\mathbf{B}_{\|} \times \hat{\mathbf{n}}_{z}\right) \cos \left(2 \omega t-\psi_{3}\right), \\
& +\frac{E_{0}^{2}}{2}\left|N_{4}\right| \mathbf{B}_{\|} \cos \left(2 \omega t-\psi_{4}\right),
\end{aligned}
$$

where the phases $\psi_{1}=\arg \left(N_{1}\right), \psi_{2}=\arg \left(N_{2}\right), \psi_{3}=\arg \left(N_{3}\right)$, $\psi_{4}=\arg \left(N_{4}\right)$ describe a time lag between the incoming light and the produced current. For the $N_{1}$ and $N_{2}$ terms, the inplane magnetic field rotates the polarization direction as in the Faraday effect [28] whereas, for the $N_{3}$ and $N_{4}$ terms, the outgoing linear polarization direction is solely determined by the parallel field (it is independent of the incoming polarization direction $\theta$ ). Note that, for unpolarized light, the $N_{1}$ and $N_{2}$ related currents are zero, but the $N_{3}$ and $N_{4}$ currents survive.

For incoming circularly polarized light Eq. (26), the $N_{3}$ and $N_{4}$-related currents are zero, and the current density is

$$
\begin{aligned}
\mathbf{J}^{(2)}= & E_{0}^{2} B_{\|}\left|N_{1}\right|\left\{\hat{\mathbf{\imath}} \cos \left(2 \omega t-\psi_{1}-\mu[\varphi-\pi / 2]\right)\right. \\
& \left.+\mu \hat{\boldsymbol{\jmath}} \sin \left(2 \omega t-\psi_{1}-\mu[\varphi-\pi / 2]\right)\right\}, \\
& +E_{0}^{2} B_{\|}\left|N_{2}\right|\left\{\hat{\mathbf{i}} \cos \left(2 \omega t-\psi_{2}-\mu \varphi-\pi\right)\right. \\
& \left.+\mu \hat{\boldsymbol{\jmath}} \sin \left(2 \omega t-\psi_{2}-\mu \varphi-\pi\right)\right\} .
\end{aligned}
$$

Thus, the generated current is also circularly polarized; the direction of the magnetic field contributes to the phase lag.
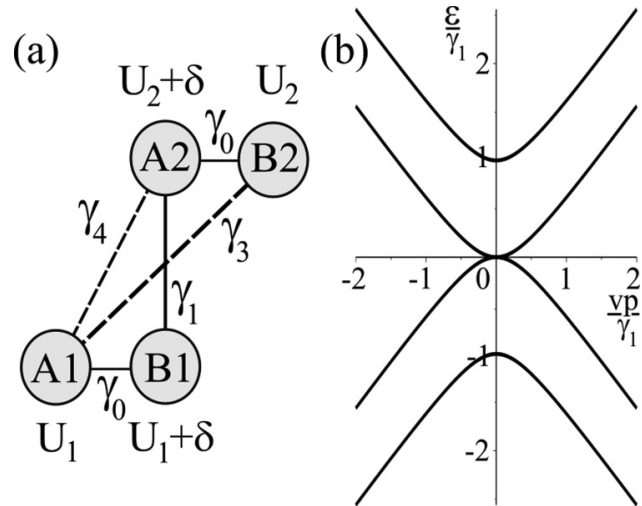

FIG. 2. (a) Schematic of the unit cell of bilayer graphene with four atoms in the unit cell, A1, B1 on the lower layer, A2, B2 on the upper layer, and relevant tight-binding parameters. (b) Schematic of the four energy bands near each $K$ point including two conduction bands and two valence bands.

\section{BILAYER GRAPHENE}

\section{A. Four-component Hamiltonian}

In order to apply the general equations for the second-order conductivities derived in Sec. III to a particular system, it is necessary to model scattering in the presence of an in-plane magnetic field in that system in order to derive the form of parameters $\Lambda_{1}$ [Eq. (19)] and $\Omega, \Omega_{c}, \Omega_{s}$ [Eq. (16)]. For bilayer graphene, this has been done previously [10] in order to model the magnetic ratchet; here we also include cyclotron resonance and second harmonic generation. We will briefly describe electronic scattering in bilayer graphene in the presence of an in-plane magnetic field but we refer the reader to [10] for further details.

Bilayer graphene has four atomic sites in the unit cell, Fig. 2(a); we label them as A1, B1 on the lower layer, and A2, B2 on the upper layer. Sites B1 and A2 lie directly below and above each other, and, as a result, their orbitals are relatively strongly coupled and these sites are referred to as "dimer" sites. We employ a Cartesian coordinate system with the graphene lying in the $x-y$ plane, $z$ in the perpendicular direction, the lower layer of the bilayer is at $z=-d / 2$, and the upper layer is at $z=d / 2$, where $d$ is the interlayer spacing. We use the tight-binding model $[14,16,39,40]$ with one $p_{z}$ orbital per site, and we take the in-plane magnetic field into account, $\mathbf{B}_{\|}=\left(B_{x}, B_{y}, 0\right)$, with a vector potential $\mathbf{A}=z\left(B_{y},-B_{x}, 0\right)$ that preserves translational symmetry in the $x-y$ graphene plane.

The vector potential enters the model through a line integral appearing in the matrix elements, for example, the matrix element for in-plane hopping between an $A$ atom at $\mathbf{R}_{A}$ and three nearest-neighbor $B$ atoms at $\mathbf{R}_{B j}, j=1,2,3$ is given by

$$
H_{A B}=-\gamma_{0} \sum_{j=1}^{3} \exp \left(i \mathbf{k} \cdot\left(\mathbf{R}_{B j}-\mathbf{R}_{A}\right)-\frac{i e}{\hbar} \int_{\mathbf{R}_{B j}}^{\mathbf{R}_{A}} \mathbf{A} \cdot \mathbf{d} \ell\right),
$$

where $\gamma_{0}$ is a tight-binding parameter and $\mathbf{k}$ is the wave vector. Two nonequivalent valleys are located at the Brillouin-zone corners ( $K$ points), wave vector $\mathbf{K}_{\xi}=\xi(4 \pi / 3 a, 0), \xi= \pm 1$, and, in the vicinity of these points, the in-plane momentum is 
$\mathbf{p}=\left(p_{x}, p_{y}, 0\right)=\hbar \mathbf{k}-\hbar \mathbf{K}_{\xi}$. Keeping linear in $\mathbf{p}$ and linear in $\mathbf{B}_{\|}$contributions, the Hamiltonian [10] in a basis of A1, B1, $\mathrm{A} 2$, B2 sites is

$$
H=\left(\begin{array}{cccc}
U_{1} & v \pi_{1}^{\dagger} & -v_{4} \pi^{\dagger} & v_{3} \pi \\
v \pi_{1} & U_{1}+\delta & \gamma_{1} & -v_{4} \pi^{\dagger} \\
-v_{4} \pi & \gamma_{1} & U_{2}+\delta & v \pi_{2}^{\dagger} \\
v_{3} \pi^{\dagger} & -v_{4} \pi & v \pi_{2} & U_{2}
\end{array}\right),
$$

where $v=\sqrt{3} a \gamma_{0} /(2 \hbar)$ represents in-plane nearest-neighbor A1-B1, A2-B2 hopping, $a$ is the lattice constant, $\gamma_{1}$ describes vertical interlayer coupling, $v_{3}=\sqrt{3} a \gamma_{3} /(2 \hbar)$ represents skew interlayer A1-B2 hopping, and $v_{4}=\sqrt{3} a \gamma_{4} /(2 \hbar)$ represents skew interlayer A1-A2, B1-B2 hopping; Fig. 2(a). The on-site energies of the two layers are characterized by $U_{1}, U_{2}$, while $\delta$ describes an energy difference between B1, A2 (dimer sites) and A1, B2 (nondimers) [16,40-43]. Complex momentum operators are labeled $\pi_{1}$ for the lower layer, $\pi_{2}$ for the upper layer, and $\pi$ for interlayer hopping:

$$
\begin{aligned}
\pi & =\xi p_{x}+i p_{y}, \\
\pi_{1} & =\xi\left(p_{x}-b_{y}\right)+i\left(p_{y}+b_{x}\right), \\
\pi_{2} & =\xi\left(p_{x}+b_{y}\right)+i\left(p_{y}-b_{x}\right),
\end{aligned}
$$

where the magnetic field, written in dimensions of momentum, is $b_{x}=e d B_{x} / 2, b_{y}=e d B_{y} / 2$.

\section{B. Two-component reduced low-energy Hamiltonian}

There are four $p_{z}$ orbitals in the unit cell and Hamiltonian (31) describes four energy bands near each $K$ point: two conduction bands, and two valence bands; Fig. 2(b). Of these, one of the conduction bands touches one valence band at the $K$ point with an approximately quadratic dispersion $\epsilon=v^{2} p^{2} / \gamma_{1}$ near zero energy $[14,16,39,40]$. The other two bands are split away from the touching point by $\pm \gamma_{1}$ because the orbitals corresponding to the $\mathrm{B} 1, \mathrm{~A} 2$ (dimer) sites are strongly coupled by $\gamma_{1}$. Thus, it is possible to represent the electronic behavior at low energy (less than $\left|\gamma_{1}\right|$ ) where there are only two bands by eliminating the components in Hamiltonian (31) related to B1, A2 (dimer) sites, resulting in a two-component Hamiltonian for the A1, B2 (nondimer) sites. This process has been described before [14,16] and, including the in-plane magnetic field [10], the two-component Hamiltonian in an A1, B2 basis is

$$
\begin{aligned}
H= & -\frac{v^{2}}{\gamma_{1}}\left(\begin{array}{cc}
0 & \left(\pi^{\dagger}\right)^{2} \\
\pi^{2} & 0
\end{array}\right)+\frac{\Delta}{2}\left[1-\frac{2 v^{2} p^{2}}{\gamma_{1}^{2}}\right]\left(\begin{array}{cc}
1 & 0 \\
0 & -1
\end{array}\right) \\
& -\frac{2 v^{2}}{\gamma_{1}}\left[\frac{v_{4}}{v}+\frac{\delta}{\gamma_{1}}\right](\mathbf{p} \times \mathbf{b})_{z}\left(\begin{array}{cc}
1 & 0 \\
0 & -1
\end{array}\right) \\
& -\frac{v v_{4} \Delta}{\gamma_{1}^{2}}\left(\begin{array}{cc}
0 & i \pi^{\dagger} \beta^{\dagger} \\
-i \pi \beta & 0
\end{array}\right),
\end{aligned}
$$

where $\beta=b_{x}+i \xi b_{y}, \beta^{\dagger}=b_{x}-i \xi b_{y}, p=|\mathbf{p}|$, and the interlayer asymmetry is $\Delta=U_{1}-U_{2}$. To derive Eq. (32), we neglected a number of contributions; further details may be found in Ref. [10]. We excluded terms that are quadratic or higher in the magnetic field, cubic or higher in $v p / \gamma_{1}$, and cubic or higher in other small parameters $v_{4} / v, \delta / \gamma_{1}, U_{1} / \gamma_{1}$, and $U_{2} / \gamma_{1}$. Note that tight-binding models that neglect parameters $v_{4}$ and $\delta$ omit the linear-in- $\mathbf{B}_{\|}$terms [44-50]. Additionally, we neglect terms that are proportional to the unit matrix in (A1, B2) space because they do not influence electronic scattering despite having a small effect on the dispersion relation.

In Eq. (32), we neglect the parameter $\gamma_{3}$. It does not produce magnetic field dependent terms in the two-component Hamiltonian so that, for the magnetic ratchet effect, it only contributes to small cross terms in the scattering probability that are higher order in small parameters than the results quoted here. It is reasonable to wonder whether $\gamma_{3}$ and interlayer asymmetry $\Delta$ could produce second-order-in-E currents for $\mathbf{B}_{\|}=0$. In fact, $\Delta$ on its own gives only isotropic terms in Eq. (32), thus it does not create coupling between harmonics, it just gives a small correction to the momentum scattering times $\tau, \tau_{2}$. The parameter $\gamma_{3}$ does produce anisotropic terms in the two-component Hamiltonian which can lead to coupling between different harmonics. However, even in the presence of finite $\Delta$, the sign of the resulting second-order-in-E current is valley dependent (like the orientation of $\gamma_{3}$-induced trigonal warping of the band structure [14]) and, after summing over both valleys, the resulting current is zero. This valley current is described in detail in Sec. V.

The first term in the Hamiltonian (32) represents chiral quasiparticles $[13,14]$ with the pseudospin direction [the relative amplitude of the electronic wave function in the lattice (A1, B2) space] in the graphene plane and fixed to the direction of the electronic momentum $\mathbf{p}=\left(p_{x}, p_{y}, 0\right)$. This term results in a quadratic dispersion $\epsilon=v^{2} p^{2} / \gamma_{1}$, and we assume that the other terms in (32) are a small perturbation with respect to this dominant one. The second term in (32) describes a gap in the spectrum [14] due to different energies $U_{1}, U_{2}$ on the two layers as characterized by interlayer asymmetry $\Delta=U_{1}-U_{2}$. Such interlayer asymmetry could be induced by an external gate voltage and, thus, the parameter $\Delta$ is, in principle, tuneable. Also, the presence of a substrate could break inversion symmetry by creating a different electrostatic potential in one layer of the bilayer as compared to the other. Substrate-induced $\Delta$ could be significant in certain circumstances: for example, it has been estimated to be $30 \mathrm{meV}$ for rippled graphene on $\mathrm{SiO}_{2}$ [51] and for graphene on hexagonal boron nitride at a small misalignment angle [52-54].

The in-plane magnetic field appears in two different terms. The first [third term in (32)] arises due to small, intrinsic lattice parameters $v_{4}$ and $\delta$, and it tends to open a gap with a direction dependent on the $z$ component of the Lorentz force $\mathbf{p} \times \mathbf{B}_{\|}$. The magnetic field also appears in the fourth term in (32); this term only appears when there is nonzero interlayer asymmetry $\Delta$. Next, we use Hamiltonian (32) to calculate the correction to the scattering rate (14) due to the in-plane field.

\section{Electron scattering}

We determine the scattering rate (14) using Hamiltonian (32) to determine eigenstates $|\mathbf{p}\rangle$ and $\left|\mathbf{p}^{\prime}\right\rangle$ in the presence of the in-plane field $\mathbf{B}_{\|}$, with scattering $\delta H$ caused by static impurities (15). The dimensionless matrix $\hat{Y}$ in $\delta H$ accounts for structure in the A1, B2 lattice degrees of freedom, that is, a possible asymmetry in the level of disorder on the two layers of the bilayer. As representative examples, we consider disorder that is symmetric with equal amounts of scattering on the two 
layers, $\hat{Y}=\hat{I}$ where $\hat{I}$ is the unit matrix, and we consider asymmetric disorder, $\hat{Y}=\left(\hat{I}+\zeta \hat{\sigma}_{z}\right) / 2$, with scattering limited to the lower $(\zeta=1)$ or upper $(\zeta=-1)$ layer. Thus, the linear-in- $\mathbf{B}_{\|}$part of the scattering rate may be written [10] in a general form as in Eq. (16) where the angle-independent factors $\Omega, \Omega_{c}, \Omega_{s}$ are

$$
\begin{aligned}
\Omega_{c}^{(\mathrm{s})}=\Omega^{(\mathrm{s})} & =\frac{\pi e d n_{i} \Delta \gamma_{1}}{2 \hbar v^{2} p^{4}}\left(\frac{\gamma_{4}}{\gamma_{0}}+\frac{\delta}{\gamma_{1}}\right)\left(1-\frac{2 v^{2} p^{2}}{\gamma_{1}^{2}}\right), \\
\Omega_{s}^{(\mathrm{s})} & =\frac{\pi e d n_{i} \Delta \gamma_{4}}{2 \hbar p^{2} \gamma_{1} \gamma_{0}},
\end{aligned}
$$

for symmetric disorder, and for asymmetric disorder

$$
\begin{aligned}
& \Omega^{(\mathrm{a})}=\frac{\pi e d n_{i}}{2 \hbar p^{2}}\left(\frac{\gamma_{4}}{\gamma_{0}}+\frac{\delta}{\gamma_{1}}\right)\left(s \zeta-\frac{\Delta}{\gamma_{1}}+\frac{\Delta \gamma_{1}}{2 v^{2} p^{2}}\right), \\
& \Omega_{c}^{(\mathrm{a})}=\Omega_{s}^{(\mathrm{a})}=0,
\end{aligned}
$$

where the density of impurities is $n_{i}=N_{i} / L^{2}$, and $s=+1$ $(s=-1)$ for states in the conduction (valence) band. Clearly, for symmetric disorder, there must be interlayer asymmetry $\Delta=U_{1}-U_{2}$ to create $\mathbf{B}_{\|}$-dependent terms, but, for asymmetric disorder, this is not necessary.

\section{Ratchet dc current $\mathrm{J}^{(0)}$ in bilayer graphene}

For bilayer graphene, we assume that the dispersion is quadratic [14], $\epsilon=v^{2} p^{2} / \gamma_{1} \equiv p^{2} /(2 m)$ with mass $m=$ $\gamma_{1} / 2 v^{2}$ and $v_{g}=2 v^{2} p / \gamma_{1}$. Then, the factor $1-p v_{g}^{\prime}-v_{g} p \tau^{\prime} / \tau$ in coefficients $M_{2}$ and $M_{3}$, Eq. (23), simplifies as $-v_{g} p \tau^{\prime} / \tau$ and these coefficients are zero unless $\tau$ is energy dependent. Overscreened Coulomb impurities in bilayer graphene act like short-range scatterers [35], $u\left(\mathbf{r}-\mathbf{R}_{j}\right)=u_{0} \delta\left(\mathbf{r}-\mathbf{R}_{j}\right)$ and $\tilde{u}\left(\mathbf{p}^{\prime}-\mathbf{p}\right)=u_{0}$, and the scattering rates Eq. (15) are

$$
\begin{gathered}
\text { symmetric disorder: } \tau^{-1}=2 \tau_{2}^{-1}=\frac{n_{i} u_{0}^{2} \gamma_{1}}{4 \hbar^{3} v^{2}}, \\
\text { asymmetric disorder: } \tau^{-1}=\tau_{2}^{-1}=\frac{n_{i} u_{0}^{2} \gamma_{1}}{8 \hbar^{3} v^{2}} .
\end{gathered}
$$

Then, if $u_{0}$ is independent of energy, so is $\tau$ and $M_{2}=M_{3}=0$. The potential is isotropic so the only nonzero harmonic is $v_{0}=$ $u_{0}^{2}$ and parameter $\Lambda_{1}$, Eq. (19), becomes $\Lambda_{1}=u_{0}^{2}\left[\Omega+\left(\Omega_{c}+\right.\right.$ $\left.\left.\Omega_{s}\right) / 2\right]$. Then, the nonlinear coefficients, $M_{1}^{(\mathrm{s})}$ for symmetric disorder, Eq. (33), and $M_{1}^{(\mathrm{a})}$ for asymmetric disorder, Eq. (34), are

$$
\begin{aligned}
M_{1}^{(\mathrm{s})}= & \frac{e^{4} d}{8 \pi \hbar^{2} m \tau} \frac{\Delta}{\gamma_{1}}\left(\frac{5 \gamma_{4}}{\gamma_{0}}+\frac{6 \delta}{\gamma_{1}}\right) \\
& \times \frac{1}{\Upsilon^{0,1} \Upsilon^{0,2}}\left(\frac{1}{\Upsilon^{1,1}}+\frac{1}{\Upsilon^{-1,1}}\right), \\
M_{1}^{(\mathrm{a})}= & -\frac{e^{4} d}{2 \pi \hbar^{2} m \tau}\left(\frac{\gamma_{4}}{\gamma_{0}}+\frac{\delta}{\gamma_{1}}\right)\left(s \zeta-\frac{\Delta}{\gamma_{1}}\right) \\
& \times \frac{1}{\Upsilon^{0,1} \Upsilon^{0,2}}\left(\frac{1}{\Upsilon^{1,1}}+\frac{1}{\Upsilon^{-1,1}}\right),
\end{aligned}
$$

which generalize the results for $\omega_{c}=0$ from Ref. [10]. Note that both coefficients change sign under $z \rightarrow-z$ inversion because of the presence of $\Delta$ (interlayer asymmetry) and $\zeta$ (asymmetric disorder), and either mechanism (interlayer asymmetry or asymmetric disorder) produces nonzero $M_{1}$.

\section{E. Second-harmonic generation $\mathrm{J}^{(2)}$ in bilayer graphene}

For quadratic dispersion and energy-independent scattering rates, $N_{3}=N_{4}=0$ and the second harmonic is given by Eq. (5) with

$$
\begin{aligned}
N_{1}^{(\mathrm{s}, \mathrm{a})}= & \frac{c^{(\mathrm{s}, \mathrm{a})} e^{4} d}{\pi \hbar^{2} m \tau} \frac{\Delta}{\epsilon}\left(\frac{\gamma_{4}}{\gamma_{0}}+\frac{\delta}{\gamma_{1}}\right) \\
& \times\left(\frac{1}{\Upsilon^{1,1} \Upsilon^{2,2} \Upsilon^{2,1}}+\frac{1}{\Upsilon^{1,-1} \Upsilon^{2,-2} \Upsilon^{2,-1}}\right), \\
N_{2}^{(\mathrm{s}, \mathrm{a})}= & \frac{i c^{(\mathrm{s}, \mathrm{a})} e^{4} d}{\pi \hbar^{2} m \tau} \frac{\Delta}{\epsilon}\left(\frac{\gamma_{4}}{\gamma_{0}}+\frac{\delta}{\gamma_{1}}\right) \\
& \times\left(\frac{1}{\Upsilon^{1,1} \Upsilon^{2,2} \Upsilon^{2,1}}-\frac{1}{\Upsilon^{1,-1} \Upsilon^{2,-2} \Upsilon^{2,-1}}\right),
\end{aligned}
$$

where the numerical factor is $c^{(\mathrm{s})}=3 / 16$ for symmetric disorder, Eq. (33), and $c^{(\mathrm{a})}=1 / 8$ for asymmetric disorder, Eq. (34). For the case of asymmetric disorder, there is no contribution that is independent of $\Delta$, i.e., asymmetric disorder on its own cannot produce the second harmonic in bilayer graphene, in contrast to the dc current $M_{1}^{(\mathrm{a})}$.

As discussed in Sec. III C, the $N_{1}$ - and $N_{2}$-related currents are zero for incoming unpolarized light, whereas for incoming linear polarization, Eq. (29), the in-plane magnetic field rotates the polarization direction as in the Faraday effect [28]. For incoming circularly polarized light, Eq. (30), the generated current is also circularly polarized and the direction of the magnetic field contributes to the phase lag.

\section{F. Discussion}

The magnitude of the ratchet current in bilayer graphene may be estimated using values of tight-binding parameters determined by infrared spectroscopy [42] (see also Ref. [55]) such as $\gamma_{0}=3.0 \mathrm{eV}, \quad \gamma_{1}=0.4 \mathrm{eV}, \quad \gamma_{4}=0.015 \mathrm{eV}, \quad \delta=$ $0.018 \mathrm{eV}$. We also use interlayer spacing $d \approx 3.3 \AA$ and mass $m \approx 0.05 m_{e}$ where $m_{e}$ is the free-electron mass. For a typical value $\tau=0.15 \mathrm{ps}[56]$ and with values $|\mathbf{E}|=10 \mathrm{kV} \mathrm{cm}^{-1}$, $|\mathbf{B}|=7 \mathrm{~T}, \omega=2.1 \times 10^{13} \mathrm{rad} \mathrm{s}^{-1}$ from recent experiment [8], we estimate that the ratchet current density for asymmetric disorder Eq. (36) is of the order of $|\mathbf{J}| \sim\left|M_{1}^{(\mathrm{a})}\right||\mathbf{B}||\mathbf{E}|^{2} \sim$ $1 \mathrm{~mA} \mathrm{~cm}{ }^{-1}$. Note, however, that these quoted experimental values violate the condition of validity of the Boltzmann equation, $e|\mathbf{E}| v_{g} \tau \ll k_{B} T$, and this should only be considered as an order of magnitude estimation. The second harmonic, Eqs. (37) and (38), is generally of the same order of magnitude, but with an additional small parameter $\Delta / \epsilon_{F}$, its precise value is not fixed because $\epsilon_{F}$ and $\Delta$ are both tunable.

A dc current may also be generated by photogalvanics or photon-drag effects [57] or, in small samples with sufficiently high mobility, by edge photogalvanics [58]. The ratchet current described here could be distinguished by its dependence on electric and magnetic field directions Eq. (22) or the frequency dependence of the $M_{1}, M_{2}, M_{3}$ coefficients, Eq. (23).

Our results apply to intraband transitions in the semiclassical regime $\epsilon_{F} \gg \omega$ (note that recent papers [19,20] consider 
second harmonic generation in bilayer graphene at higher frequencies). For Fermi energy $\epsilon_{F}=100 \mathrm{meV}$, say, the frequency corresponding to $\hbar \omega=100 \mathrm{meV}$ is $\omega \approx 150 \times 10^{12} \mathrm{rad} \mathrm{s}^{-1}$ or linear frequency $f \approx 24 \mathrm{THz}$. For $\omega_{c}=e B_{\perp} / m$ with $m \approx$ $0.05 m_{e}$ for bilayer graphene, where $m_{e}$ is the free-electron mass, a perpendicular field $B_{\perp}=1 \mathrm{~T}$ corresponds to $\omega_{c} \approx$ $3.5 \times 10^{12} \mathrm{rad} \mathrm{s}^{-1}$. Then, the cyclotron resonance condition $\omega=\omega_{c}$ corresponds to linear frequency of light $f \approx 0.56 \mathrm{THz}$ (the corresponding energy scale is $\hbar \omega \approx 15 \mathrm{meV}$ ) which is well within the semiclassical regime considered here.

\section{VALLEY CURRENTS IN BILAYER GRAPHENE FOR $B_{\|}=0$ DUE TO TRIGONAL WARPING}

Here we consider second-order-in-electric-field currents that occur when $\mathbf{B}_{\|}=0$ due to the presence of skew interlayer A1-B2 hopping described by parameter $\gamma_{3}$, Eq. (31). These are valley currents: the sign of the current per valley depends on the valley index $(\xi= \pm 1)$ and the total current, summed over both valleys, is zero unless there is an additional source of valley polarization [19,20,26,59-62]. The two-component Hamiltonian (32) is modified as

$$
\begin{aligned}
H= & -\frac{v^{2}}{\gamma_{1}}\left(\begin{array}{cc}
0 & \left(\pi^{\dagger}\right)^{2} \\
\pi^{2} & 0
\end{array}\right)+v_{3}\left(\begin{array}{cc}
0 & \pi \\
\pi^{\dagger} & 0
\end{array}\right) \\
& +\frac{\Delta}{2}\left[1-\frac{2 v^{2} p^{2}}{\gamma_{1}^{2}}\right]\left(\begin{array}{cc}
1 & 0 \\
0 & -1
\end{array}\right),
\end{aligned}
$$

where $v_{3}=\sqrt{3} a \gamma_{3} /(2 \hbar)$. The presence of $\gamma_{3}$ creates trigonal warping of the electron band structure [14-16] in which the Fermi circle around each valley assumes a triangular distortion, with the orientation of the distortion being opposite in the two valleys.

As in Sec. IV, we assume that the first term in the Hamiltonian (39) dominates, and the other terms are small with respect to it, i.e., $v^{2} p^{2} / \gamma_{1} \gg\left\{v_{3} p, \Delta\right\}$. Interlayer asymmetry $\Delta$ has no angular dependence in (39) and it does not create coupling between harmonics, Eq. (18), it just gives a small correction to the momentum scattering times $\tau, \tau_{2}$ which we neglect. The parameter $\gamma_{3}$, however, does produce coupling between different harmonics. To lowest order in $\gamma_{3}$ the relevant correction to the scattering rate for symmetric disorder, $\hat{Y}=\hat{I}$, is

$$
\begin{aligned}
\delta W_{\mathbf{p}^{\prime} \mathbf{p}}^{(\mathrm{s})}= & \xi \frac{\pi}{\hbar} \frac{n_{i}}{L^{2}}\left|\tilde{u}\left(\mathbf{p}^{\prime}-\mathbf{p}\right)\right|^{2} \delta\left(\epsilon_{\mathbf{p}}-\epsilon_{\mathbf{p}^{\prime}}\right) \\
& \times \frac{v_{3} \gamma_{1}}{v^{2} p}\left\{\cos \left[2\left(\phi^{\prime}-\phi\right)\right]\left[\cos (3 \phi)+\cos \left(3 \phi^{\prime}\right)\right]\right. \\
& \left.-2 \cos \left[\frac{1}{2}\left(\phi^{\prime}-\phi\right)\right] \cos \left[\frac{3}{2}\left(\phi^{\prime}+\phi\right)\right]\right\},
\end{aligned}
$$

and for asymmetric disorder, $\hat{Y}=\left(\hat{I}+\zeta \hat{\sigma}_{z}\right) / 2$, it is

$$
\begin{aligned}
\delta W_{\mathbf{p}^{\prime} \mathbf{p}}^{(\mathrm{a})}= & s \zeta \frac{\pi n_{i} \gamma_{1} \Delta}{4 \hbar v^{2} L^{2}}\left(1-\frac{2 v^{2} p^{2}}{\gamma_{1}^{2}}\right)\left|\tilde{u}\left(\mathbf{p}^{\prime}-\mathbf{p}\right)\right|^{2} \delta\left(\epsilon_{\mathbf{p}}-\epsilon_{\mathbf{p}^{\prime}}\right) \\
& \times\left[\frac{1}{p^{2}}+\frac{1}{\left(p^{\prime}\right)^{2}}+\frac{\xi v_{3} \gamma_{1}}{v^{2} p^{3}} \cos (3 \phi)+\frac{\xi v_{3} \gamma_{1}}{v^{2}\left(p^{\prime}\right)^{3}} \cos \left(3 \phi^{\prime}\right)\right] .
\end{aligned}
$$

Following the harmonic expansion, these terms lead to coupling between different harmonics, Eq. (18), with

$$
\delta S_{j}^{(\ell)}=C_{j-3} f_{j-3}^{(\ell)}+C_{-j-3} f_{j+3}^{(\ell)},
$$

where, for symmetric or asymmetric disorder,

$$
\begin{aligned}
C_{j}^{(\mathrm{s})}= & \xi \frac{\pi n_{i} \Gamma v_{3} \gamma_{1}}{4 \hbar v^{2} p} \\
& \times\left(v_{j+5}-v_{5}-v_{j+2}+v_{j-2}-v_{j+1}+v_{1}\right), \\
C_{j}^{(\mathrm{a})} & =s \xi \zeta \frac{\pi n_{i} \Gamma v_{3} \Delta \gamma_{1}^{2}}{4 \hbar v^{4} p^{3}}\left(1-\frac{2 v^{2} p^{2}}{\gamma_{1}^{2}}\right)\left(v_{j}-v_{0}\right) .
\end{aligned}
$$

The dc current per valley $\mathbf{J}^{(0)}$ and the second harmonic current per valley $\mathbf{J}^{(2)}$ may be written as

$$
\begin{aligned}
& \mathbf{J}^{(0)}=\left(\hat{\mathbf{i}} \Theta_{1}-\hat{\boldsymbol{j}} \Theta_{2}\right) \operatorname{Re} Q-\left(\hat{\mathbf{i}} \Theta_{2}+\hat{\boldsymbol{j}} \Theta_{1}\right) \operatorname{Im} Q, \\
& \mathbf{J}^{(2)}=2 \operatorname{Re}\left\{\left[S_{1}\left(\hat{\mathbf{i}} \Theta_{4}-\hat{\boldsymbol{j}} \Theta_{5}\right)+S_{2}\left(\hat{\mathbf{i}} \Theta_{5}+\hat{\boldsymbol{j}} \Theta_{4}\right)\right] e^{-2 i \omega t}\right\},
\end{aligned}
$$

where $\Theta_{1}=\left(\left|E_{x}\right|^{2}-\left|E_{y}\right|^{2}\right), \Theta_{2}=\left(E_{x} E_{y}^{*}+E_{y} E_{x}^{*}\right), \Theta_{4}=$ $\left(E_{x}^{2}-E_{y}^{2}\right)$, and $\Theta_{5}=2 E_{x} E_{y}$. For a degenerate electron gas, $\epsilon_{F} \gg k_{B} T$, the coefficients $Q, S_{1}, S_{2}$ are given by

$$
\begin{aligned}
& Q=-\frac{g e^{3} v_{g}}{8 \pi \hbar^{2}}\left(\frac{1}{\Upsilon^{1,-1}}+\frac{1}{\Upsilon^{-1,-1}}\right) \\
& \times\left[\frac{2 C_{-2}}{\Upsilon^{0,1} \Upsilon^{0,-2}}+p\left(\frac{C_{-2} v_{g}}{\Upsilon^{0,1} \Upsilon^{0,-2}}\right)^{\prime}\right] \text {, } \\
& +\frac{g e^{3} C_{-1} v_{g}}{8 \pi \hbar^{2}}\left(\frac{1}{\Upsilon^{1,2} \Upsilon^{1,-1}}+\frac{1}{\Upsilon^{-1,2} \Upsilon^{-1,-1}}\right) \\
& \times\left[\frac{1}{\Upsilon^{0,1}}-p\left(\frac{v_{g}}{\Upsilon^{0,1}}\right)^{\prime}\right] \text {, } \\
& S_{1}=-\frac{g e^{3} v_{g}}{16 \pi \hbar^{2}}\left[\frac{2 C_{-2}}{\Upsilon^{1,1} \Upsilon^{2,-1} \Upsilon^{2,2}}+\frac{2 C_{-2}}{\Upsilon^{1,-1} \Upsilon^{2,1} \Upsilon^{2,-2}}\right. \\
& +\frac{p}{\Upsilon^{1,1}}\left(\frac{v_{g} C_{-2}}{\Upsilon^{2,-1} \Upsilon^{2,2}}\right)^{\prime}+\frac{p}{\Upsilon^{1,-1}}\left(\frac{v_{g} C_{-2}}{\Upsilon^{2,1} \Upsilon^{2,-2}}\right)^{\prime} \\
& -\frac{C_{-1}}{\Upsilon^{1,1} \Upsilon^{1,-2} \Upsilon^{2,-1}}-\frac{C_{-1}}{\Upsilon^{1,-1} \Upsilon^{1,2} \Upsilon^{2,1}} \\
& \left.+\frac{C_{-1} p}{\Upsilon^{1,1} \Upsilon^{1,-2}}\left(\frac{v_{g}}{\Upsilon^{2,-1}}\right)^{\prime}+\frac{C_{-1} p}{\Upsilon^{1,-1} \Upsilon^{1,2}}\left(\frac{v_{g}}{\Upsilon^{2,1}}\right)^{\prime}\right] \text {, } \\
& S_{2}=\frac{i g e^{3} v_{g}}{16 \pi \hbar^{2}}\left[\frac{2 C_{-2}}{\Upsilon^{1,1} \Upsilon^{2,-1} \Upsilon^{2,2}}-\frac{2 C_{-2}}{\Upsilon^{1,-1} \Upsilon^{2,1} \Upsilon^{2,-2}}\right. \\
& +\frac{p}{\Upsilon^{1,1}}\left(\frac{v_{g} C_{-2}}{\Upsilon^{2,-1} \Upsilon^{2,2}}\right)^{\prime}-\frac{p}{\Upsilon^{1,-1}}\left(\frac{v_{g} C_{-2}}{\Upsilon^{2,1} \Upsilon^{2,-2}}\right) \\
& -\frac{C_{-1}}{\Upsilon^{1,1} \Upsilon^{1,-2} \Upsilon^{2,-1}}+\frac{C_{-1}}{\Upsilon^{1,-1} \Upsilon^{1,2} \Upsilon^{2,1}} \\
& \left.+\frac{C_{-1} p}{\Upsilon^{1,1} \Upsilon^{1,-2}}\left(\frac{v_{g}}{\Upsilon^{2,-1}}\right)^{\prime}-\frac{C_{-1} p}{\Upsilon^{1,-1} \Upsilon^{1,2}}\left(\frac{v_{g}}{\Upsilon^{2,1}}\right)^{\prime}\right],
\end{aligned}
$$

where $(\ldots)^{\prime} \equiv \partial(\ldots) / \partial \epsilon, g=2$ for spin and all parameters are evaluated on the Fermi surface. Assuming isotropic, shortrange impurities and scattering times $\tau, \tau_{2}$ independent of energy (as in Sec. IVD), the coefficients $Q, S_{1}, S_{2}$ simplify 
as

$$
\begin{aligned}
Q^{(\mathrm{s}, \mathrm{a})} & =\xi r^{(\mathrm{s}, \mathrm{a})} \frac{e^{3} v_{3}}{4 \pi \hbar^{2} \tau} \frac{1}{\Upsilon^{0,1} \Upsilon^{0,-2}}\left(\frac{1}{\Upsilon^{1,-1}}+\frac{1}{\Upsilon^{-1,-1}}\right), \\
S_{1}^{(\mathrm{s}, \mathrm{a})} & =\xi r^{(\mathrm{s}, \mathrm{a})} \frac{e^{3} v_{3}}{8 \pi \hbar^{2} \tau}\left(\frac{1}{\Upsilon^{1,1} \Upsilon^{2,-1} \Upsilon^{2,2}}+\frac{1}{\Upsilon^{1,-1} \Upsilon^{2,1} \Upsilon^{2,-2}}\right), \\
S_{2}^{(\mathrm{s}, \mathrm{a})} & =-\xi r^{(\mathrm{s}, \mathrm{a})} \frac{i e^{3} v_{3}}{8 \pi \hbar^{2} \tau}\left(\frac{1}{\Upsilon^{1,1} \Upsilon^{2,-1} \Upsilon^{2,2}}-\frac{1}{\Upsilon^{1,-1} \Upsilon^{2,1} \Upsilon^{2,-2}}\right),
\end{aligned}
$$

where the factor $r^{(\mathrm{s})}=1$ for symmetric disorder and $r^{(\mathrm{a})}=$ $-s \zeta \Delta / \gamma_{1}$ for asymmetric disorder.

The form of the valley currents, Eqs. (43) and (44), is consistent with threefold rotational symmetry (rotations around the $z$ axis by $2 \pi / 3$ ). Terms $\operatorname{Re} Q$ and $S_{1}$ are even functions of $\hat{\mathbf{n}}_{z} \cdot \mathbf{B}_{\perp}$, whereas Im $Q$ and $S_{2}$ are odd functions, thus they are zero for $\mathbf{B}_{\perp}=0$. The dc valley current is only nonzero for incoming linearly polarized light, Eq. (24), for which the valley currents may be written as

$$
\begin{aligned}
\mathbf{J}^{(0)}= & \frac{1}{4} E_{0}^{2}|Q|\left[\hat{\mathbf{i}} \cos \left(2 \theta+\Pi_{Q}\right)-\hat{\boldsymbol{j}} \sin \left(2 \theta+\Pi_{Q}\right)\right], \\
\mathbf{J}^{(2)}= & \frac{1}{2} E_{0}^{2}\left|S_{1}\right| \cos \left(2 \omega t-\Pi_{1}\right)(\hat{\mathbf{i}} \cos 2 \theta-\hat{\boldsymbol{j}} \sin 2 \theta) \\
& +\frac{1}{2} E_{0}^{2}\left|S_{2}\right| \cos \left(2 \omega t-\Pi_{2}\right)(\hat{\mathbf{i}} \sin 2 \theta+\hat{\boldsymbol{j}} \cos 2 \theta),
\end{aligned}
$$

where $\Pi_{Q}=\arg (Q), \Pi_{1}=\arg \left(S_{1}\right)$, and $\Pi_{2}=\arg \left(S_{2}\right)$. This indicates that the incoming polarization angle $\theta$ dictates the direction of outgoing dc current and the polarization of the second harmonic. For incoming circularly polarized light, Eq. (26), the dc current is zero. For the second harmonic

$$
\begin{aligned}
\mathbf{J}^{(2)}= & E_{0}^{2}\left|S_{1}\right|\left\{\hat{\mathbf{i}} \cos \left(2 \omega t-\Pi_{1}\right)-\mu \hat{\boldsymbol{j}} \sin \left(2 \omega t-\Pi_{1}\right)\right\}, \\
& +E_{0}^{2}\left|S_{2}\right|\left\{\hat{\mathbf{i}} \cos \left(2 \omega t-\Pi_{2}-\mu \pi / 2\right)\right. \\
& \left.-\mu \hat{\boldsymbol{\jmath}} \sin \left(2 \omega t-\Pi_{2}-\mu \pi / 2\right)\right\} .
\end{aligned}
$$

Thus, the generated current is also circularly polarized, but the sense of the polarization is reversed as compared to the incoming light.

The valley currents display cyclotron resonances at the same frequencies as the magnetic ratchet: $\omega_{c}=\omega$ for the dc current and $\omega_{c}=\omega$ and $\omega_{c}=2 \omega$ for the second harmonic. The valley current is much larger than the magnetic ratchet current per valley, typically by a factor of $m v_{3} /\left[|\mathbf{B}| e d\left(\gamma_{4} / \gamma_{0}+\delta / \gamma_{1}\right)\right] \sim$ 1500 for $|\mathbf{B}|=1 \mathrm{~T}$, although, obviously, the total current is zero when summed over both valleys. Although we have considered only linear powers of $\gamma_{3}$, a similar qualitative picture holds for higher powers. Even powers of $\gamma_{3}$ would not appear with the valley index, but they are only capable of coupling harmonics with values of the angular index $j$ that differ by an even number. Hence, they do not produce second-order-in-E currents. Odd powers of $\gamma_{3}$ can couple harmonics with values of the angular index $j$ that differ by an odd number [as in Eq. (40)], thus yielding second-order-in-E currents, but they always appear with the valley index, giving a total current of zero.

\section{CONCLUSIONS}

As detailed in Sec. III, we have determined the dc current, Eq. (22), and the second harmonic generation, Eq. (28), for the magnetic ratchet in the semiclassical regime $\left(\epsilon_{F} \gg \hbar \omega\right)$ in a two-dimensional electron system. These results apply to systems with arbitrary, isotropic dispersion, and energydependent scattering rates. For the particular case of bilayer graphene, we assume a perfectly quadratic dispersion relation $\epsilon=p^{2} / 2 m$ and relaxation rates that are independent of energy to produce simplified expressions for the dc current, Eqs. (1), (35), and (36), and second harmonic generation, Eqs. (5), (37), and (38). We take into account inversion symmetry breaking by disorder and by interlayer asymmetry; the latter may potentially be induced using an external gate and is thus tunable. In the presence of a tilted field, we find that the dc current has a resonance at $\omega_{c}=\omega$ but that the current value is actually largest at $\omega_{c}=0$. For the second harmonic, however, resonances at $\omega_{c}=\omega$ and $\omega_{c}=2 \omega$ generally produce currents significantly greater than that at $\omega_{c}=0$.

\section{ACKNOWLEDGMENTS}

We thank S. D. Ganichev and S. Slizovskiy for useful discussions. This work was funded by the European Union Grant Agreement No. 696656 Graphene Flagship, the ERC Synergy Grant Hetero2D, and EPSRC Grant No. EP/N010345/1.
[1] V. I. Fal'ko, Sov. Phys. Solid State 31, 561 (1989) [Fiz. Tverd. Tela 31, 29 (1989)].

[2] S. A. Tarasenko, Phys. Rev. B 77, 085328 (2008).

[3] S. A. Tarasenko, Phys. Rev. B 83, 035313 (2011).

[4] S. D. Ganichev, S. A. Tarasenko, J. Karch, J. Kamann, and Z. D. Kvon, J. Phys.: Condens. Matter 26, 255802 (2014).

[5] G. V. Budkin and L. E. Golub, Phys. Rev. B 90, 125316 (2014).

[6] G. V. Budkin and S. A. Tarasenko, Phys. Rev. B 93, 075306 (2016).

[7] A. V. Nalitov, L. E. Golub, and E. L. Ivchenko, Phys. Rev. B 86, 115301 (2012).

[8] C. Drexler, S. A. Tarasenko, P. Olbrich, J. Karch, M. Hirmer, F. Müller, M. Gmitra, J. Fabian, R. Yakimova, S. Lara-Avila, S. Kubatkin, M. Wang, R. Vajtai, P. M. Ajayan, J. Kono, and S. D. Ganichev, Nat. Nanotechnol. 8, 104 (2013).
[9] P. Olbrich, J. Kamann, M. König, J. Munzert, L. Tutsch, J. Eroms, D. Weiss, M.-H. Liu, L. E. Golub, E. L. Ivchenko, V. V. Popov, D. V. Fateev, K. V. Mashinsky, F. Fromm, Th. Seyller, and S. D. Ganichev, Phys. Rev. B 93, 075422 (2016).

[10] N. Kheirabadi, E. McCann, and V. I. Fal'ko, Phys. Rev. B 94, 165404 (2016).

[11] S. A. Mikhailov, Europhys. Lett. 79, 27002 (2007).

[12] M. M. Glazov and S. D. Ganichev, Phys. Rep. 535, 101 (2014).

[13] K. S. Novoselov, E. McCann, S. V. Morozov, V. I. Fal'ko, M. I. Katsnelson, U. Zeitler, D. Jiang, F. Schedin, and A. K. Geim, Nat. Phys. 2, 177 (2006).

[14] E. McCann and V. I. Fal'ko, Phys. Rev. Lett. 96, 086805 (2006).

[15] E. McCann, D. S. L. Abergel, and V. I. Fal'ko, Solid State Commun. 143, 110 (2007).

[16] E. McCann and M. Koshino, Rep. Prog. Phys. 76, 056503 (2013). 
[17] A. V. Rozhkov, A. O. Sboychakov, A. L. Rakhmanov, and F. Nori, Phys. Rep. 648, 1 (2016).

[18] E. McCann, Phys. Rev. B 74, 161403(R) (2006).

[19] S. Wu, L. Mao, A. M. Jones, W. Yao, C. Zhang, and X. Xu, Nano Lett. 12, 2032 (2012).

[20] S. J. Brun and T. G. Pedersen, Phys. Rev. B 91, 205405 (2015).

[21] J. J. Dean and H. M. van Driel, Appl. Phys. Lett. 95, 261910 (2009)

[22] J. J. Dean and H. M. van Driel, Phys. Rev. B 82, 125411 (2010)

[23] M. M. Glazov, JETP Lett. 93, 366 (2011).

[24] S. A. Mikhailov, Phys. Rev. B 84, 045432 (2011).

[25] A. Y. Bykov, T. V. Murzina, M. G. Rybin, and E. D. Obraztsova, Phys. Rev. B 85, 121413(R) 2012 .

[26] L. E. Golub and S. A. Tarasenko, Phys. Rev. B 90, 201402(R) (2014).

[27] K.-H. Lin, S.-W. Weng, P.-W. Lyu, T.-R. Tsai, and W.-B. Su, Appl. Phys. Lett. 105, 151605 (2014).

[28] F. L. Pedrotti and L. S. Pedrotti, Introduction to Optics, 2nd ed. (Prentice-Hall, Englewood Cliffs, NJ, 1993).

[29] Y. R. Shen, The Principles of Nonlinear Optics (John Wiley and Sons, Hoboken, NJ, 2003).

[30] R. W. Boyd, Nonlinear Optics, 3rd ed. (Academic Press, Burlington, MA, 2008).

[31] P. Olbrich, C. Zoth, P. Vierling, K.-M. Dantscher, G. V. Budkin, S. A. Tarasenko, V. V. Bel'kov, D. A. Kozlov, Z. D. Kvon, N. N. Mikhailov, S. A. Dvoretsky, and S. D. Ganichev, Phys. Rev. B 87, 235439 (2013).

[32] K.-M. Dantscher, D. A. Kozlov, P. Olbrich, C. Zoth, P. Faltermeier, M. Lindner, G. V. Budkin, S. A. Tarasenko, V. V. Bel'kov, Z. D. Kvon, N. N. Mikhailov, S. A. Dvoretsky, D. Weiss, B. Jenichen, and S. D. Ganichev, Phys. Rev. B 92, 165314 (2015).

[33] T. Ando, J. Phys. Soc. Jpn. 75, 074716 (2006).

[34] T. Heinzel, Mesoscopic Electronics in Solid State Nanostructures, 3rd ed. (Wiley-VCH, Weinheim, 2010).

[35] S. Adam and S. Das Sarma, Phys. Rev. B 77, 115436 (2008).

[36] K. Nomura and A. H. MacDonald, Phys. Rev. Lett. 98, 076602 (2007).

[37] E. H. Hwang, S. Adam, and S. Das Sarma, Phys. Rev. Lett. 98, 186806 (2007).

[38] Coefficients $M_{1}, M_{2}, M_{3}$ in Eq. (22) are related to $M_{0}, M_{L}, M_{C}$ in [6] by $M_{1}=M_{L}, M_{2}=M_{0}, M_{3}=i M_{C}$. These choices are made to be consistent with earlier works $[3,10]$ and to ensure the coefficients are real for $\omega_{c}=0$.

[39] F. Guinea, A. H. Castro Neto, and N. M. R. Peres, Phys. Rev. B 73, 245426 (2006).

[40] J. Nilsson, A. H. Castro Neto, F. Guinea, and N. M. R. Peres, Phys. Rev. B 78, 045405 (2008).
[41] M. S. Dresselhaus and G. Dresselhaus, Adv. Phys. 51, 1 (2002).

[42] L. M. Zhang, Z. Q. Li, D. N. Basov, M. M. Fogler, Z. Hao, and M. C. Martin, Phys. Rev. B 78, 235408 (2008).

[43] Z. Q. Li, E. A. Henriksen, Z. Jiang, Z. Hao, M. C. Martin, P. Kim, H. L. Stormer, and D. N. Basov, Phys. Rev. Lett. 102, 037403 (2009).

[44] S. S. Pershoguba and V. M. Yakovenko, Phys. Rev. B 82, 205408 (2010).

[45] Y.-H. Hyun, Y. Kim, C. Sochichiu, and M.-Y. Choi, J. Phys.: Condens. Matter 24, 045501 (2012).

[46] N. A. Goncharuk and L. Smrčka, J. Phys.: Condens. Matter 24, 185503 (2012).

[47] B. Roy and K. Yang, Phys. Rev. B 88, 241107(R) (2013).

[48] W.-Y. He, Y. Su, M. Yang, and L. He, Phys. Rev. B 89, 125418 (2014).

[49] M. Van der Donck, F. M. Peeters, and B. Van Duppen, Phys. Rev. B 93, 115423 (2016).

[50] M. Van der Donck, F. M. Peeters, and B. Van Duppen, Phys. Rev. B 93, 247401 (2016).

[51] E.-A. Kim and A. H. Castro Neto, Europhys. Lett. 84, 57007 (2008).

[52] M. Yankowitz, J. Xue, D. Cormode, J. D. Sanchez-Yamagishi, K. Watanabe, T. Taniguchi, P. Jarillo-Herrero, P. Jacquod, and B. J. LeRoy, Nat. Phys. 8, 382 (2012).

[53] P. Moon and M. Koshino, Phys. Rev. B 90, 155406 (2014).

[54] X. Chen, J. R. Wallbank, M. Mucha-Kruczyński, E. McCann, and V. I. Fal'ko, Phys. Rev. B 94, 045442 (2016).

[55] A. B. Kuzmenko, I. Crassee, D. van der Marel, P. Blake, and K. S. Novoselov, Phys. Rev. B 80, 165406 (2009).

[56] R. V. Gorbachev, F. V. Tikhonenko, A. S. Mayorov, D. W. Horsell, and A. K. Savchenko, Phys. Rev. Lett. 98, 176805 (2007).

[57] C. Jiang, V. A. Shalygin, V. Yu. Panevin, S. N. Danilov, M. M. Glazov, R. Yakimova, S. Lara-Avila, S. Kubatkin, and S. D. Ganichev, Phys. Rev. B 84, 125429 (2011).

[58] J. Karch, C. Drexler, P. Olbrich, M. Fehrenbacher, M. Hirmer, M. M. Glazov, S. A. Tarasenko, E. L. Ivchenko, B. Birkner, J. Eroms, D. Weiss, R. Yakimova, S. Lara-Avila, S. Kubatkin, M. Ostler, T. Seyller, and S. D. Ganichev, Phys. Rev. Lett. 107, 276601 (2011).

[59] A. Rycerz, J. Tworzydło, and C. W. J. Beenakker, Nat. Phys. 3, 172 (2007).

[60] D. Xiao, W. Yao, and Q. Niu, Phys. Rev. Lett. 99, 236809 (2007).

[61] J. Wang, K. S. Chan, and Z. Lin, Appl. Phys. Lett. 104, 013105 (2014).

[62] H. Yu, Y. Wu, G.-B. Liu, X. Xu, and W. Yao, Phys. Rev. Lett. 113, 156603 (2014). 